\title{
Bayesian Model Averaging in the Presence of Structural Breaks*
}

\author{
Francesco Ravazzolo, Richard Paap, \\ Dick van Dijk ${ }^{\dagger}$ and Philip Hans Franses \\ Econometric Institute \\ Erasmus University Rotterdam
}

\section{ECONOMETRIC Institute RePort EI 2006-33}

August 2006

\begin{abstract}
This paper develops a return forecasting methodology that allows for instability in the relationship between stock returns and predictor variables, for model uncertainty, and for parameter estimation uncertainty. The predictive regression specification that is put forward allows for occasional structural breaks of random magnitude in the regression parameters, and for uncertainty about the inclusion of forecasting variables, and about the parameter values by employing Bayesian Model Averaging. The implications of these three sources of uncertainty, and their relative importance, are investigated from an active investment management perspective. It is found that the economic value of incorporating all three sources of uncertainty is considerable. A typical investor would be willing to pay up to several hundreds of basis points annually to switch from a passive buy-and-hold strategy to an active strategy based on a return forecasting model that allows for model and parameter uncertainty as well as structural breaks in the regression parameters.
\end{abstract}

Keywords: Stock return predictability, model uncertainty, Bayesian model averaging, structural breaks, portfolio selection.

JEL Classification: G11, G12, C11

\footnotetext{
*We thank Marno Verbeek and Wessel Marquering for providing their data set.

${ }^{\dagger}$ Corresponding author: Econometric Institute, Erasmus University Rotterdam, P.O. Box 1738, NL-3000 DR Rotterdam, The Netherlands, e-mail: djvandijk@few.eur.nl
} 


\section{Introduction}

A growing body of empirical evidence suggests the presence of a certain (albeit modest) level of predictability in stock returns. Several financial and macro-economic variables have been reported as being useful predictors of future stock returns, including interest rates and different interest rate spreads (such as the yield spread, term spread and credit spread), as well as valuation ratios such as the dividend yield and the price-earnings ratio. There is, however, little consensus about which variables really are the relevant predictor variables that should enter a successful return forecasting model. Put differently, an investor who intends to use a predictive regression to forecast future stock returns faces model uncertainty.

At the same time, recent studies demonstrate that the relationship between stock returns and predictor variables is not stable over time, see Pesaran and Timmermann (2002), among others. Important political and economic events, such as changes in monetary policy, oil crises and recessions fundamentally change the economic environment including financial markets. In terms of predictive regressions for stock returns, an investor should take into account that parameters exhibit occasional structural breaks.

A third related issue that investors have to cope with is the fact that parameters in return forecasting model are estimated using historical data, implying the presence of parameter (estimation) uncertainty.

While model uncertainty and structural breaks in the context of return prediction models have been studied in isolation, attempts to consider both features simultaneously are very rare, but see Pettenuzzo and Timmermann (2005). In this section we develop the return forecasting methodology that allows for instability in the relationship between stock returns and predictor variables, for model uncertainty, and for parameter estimation uncertainty simultaneously. On the one hand, the predictive regression specification that we put forward allows for occasional structural breaks of random magnitude in the regression parameters. On the other hand, we allow for uncertainty about the inclusion of the forecasting variables in the model and about the parameter values by employing Bayesian model averaging.

The paper proceeds as follows. In Section 2 we describe our methodology, and put forward the predictive regression specification that incorporates all three relevant sources of uncertainty together. Given that the Bayesian analysis of our model is non-standard, we provide a detailed description of the prior specification and the simulation of the posterior distributions. In Section 3 we report results from an empirical application of the approach developed in Section 2 to predicting US stock 
returns using a set of 11 financial and macro-economic predictor variables. We find that over the period 1966-2005, several structural breaks occurred in the relationship between US stock returns and predictor variables such as the dividend yield and interest rates. These changes appear to be caused by important events such as the oil crisis, changes in monetary policy, the October 1987 stock market crash, and the internet bubble at the end of the 1990s. The economic value of incorporating the different sources of uncertainty in investment decisions in real-time is assessed in Section 4 , by means of an ex-ante recursive forecasting experiment. We find that a typical investor would be willing to pay up to several hundreds of basis points annually to switch from a passive buy-and-hold strategy to an active strategy based on a return forecasting model that allows for model and parameter uncertainty as well as structural breaks in the regression parameters. Section 5 concludes.

\section{Methodology}

In this section we develop the return forecasting methodology that allows for instability in the relationship between stock returns and predictor variables, for model uncertainty, and for parameter estimation uncertainty simultaneously. On the one hand, the predictive regression specification that we put forward allows for occasional structural breaks of random magnitude in the regression parameters. On the other hand, we allow for uncertainty about the inclusion of the forecasting variables in the model and about the parameter values by employing Bayesian Model Averaging (BMA). Given that the Bayesian analysis of our model is non-standard, we provide a detailed description of the prior specification and the simulation of the posterior distributions. Finally, we conclude with some remarks on possible uses of the posterior results, including forecasting future returns. How to use those in active investment strategies is discussed in Section 4.

\subsection{The Model}

Let $r_{t}$ denote the stock return in excess of the risk-free rate during period $t$, and let $x_{t}=\left(x_{1 t}, x_{2 t}, \ldots, x_{k t}\right)^{\prime}$ denote a vector of $k$ predictor variables (which are observed at the beginning of period $t$ ) for $t=1, \ldots, T$. The benchmark model in the literature for predicting stock returns is the standard linear regression model

$$
r_{t}=\beta_{0}+\sum_{j=1}^{k} \beta_{j} x_{j t}+\varepsilon_{t}
$$


where $\varepsilon_{t} \sim N\left(0, \sigma^{2}\right)$. Two crucial assumptions (among others) underlying the linear regression model are, first, that the set of relevant predictor variables $x_{t}$ is given and fixed, and second that the regression parameters $\beta=\left(\beta_{0}, \beta_{1}, \ldots, \beta_{k}\right)$ are constant over time. Both assumptions are questionable in empirical practice, and extensions of the model that drop either of the two assumptions have been developed in recent years. These are briefly discussed first, before we introduce our general model that allows for both uncertainty about the relevant predictor variables and for possible structural breaks in the regression parameters.

First, the fact that the set of predictor variables $x_{t}$ in (1) is given and fixed $a$ priori is unrealistic, in the sense that the investor rarely knows with certainty which particular forecasting variables are the relevant ones to include. Avramov (2002) and Cremers (2002) have analyzed this issue of model uncertainty, advocating the use of Bayesian model averaging where all $2^{k}$ possible models are considered (assuming the intercept is always included in the model) and averaged according to their posterior probabilities.

A possible way to represent model uncertainty in the linear regression is by means of a latent binary random variable $s_{j}=0,1$ determining the inclusion of $x_{j t}$ in the model, with $\operatorname{Pr}\left[s_{j}=1\right]=\lambda_{j}$ for $j=1, \ldots, k$. The return forecasting model with uncertainty about the relevant predictor variables (but with constant parameters) then is given by

$$
r_{t}=\beta_{0}+\sum_{j=1}^{k} s_{j} \beta_{j} x_{j t}+\varepsilon_{t} .
$$

The $k s_{j}$ variables can be summarized in a $k$-dimensional vector $S=\left(s_{1}, \ldots, s_{k}\right)$. The vector $S$ can take $2^{k}$ different values resulting in $2^{k}$ possible different regression models. Model selection is therefore defined in terms of variable selection, see George and McCulloch (1993) and Kuo and Mallick (1998). We denote each model by the index $i=\left(s_{1}, \ldots, s_{k}\right)_{2}$. Note the intercept parameter $\beta_{0}$ is always included in the model, as typically assumed.

Second, as discussed in the introduction, there is abundant empirical evidence showing that the relationship between stock returns and typical predictor variables such as the dividend yield is not stable over time, implying that the assumption of constant regression parameters $\beta_{j}$ as in (1) is invalid.

There are several ways to extend the linear regression model in order to capture parameter instability. An attractive flexible specification that allows for occasional 
structural breaks in the regression parameters is as follows:

$$
r_{t}=\beta_{0 t}+\sum_{j=1}^{k} \beta_{j t} x_{j t}+\varepsilon_{t},
$$

where $\beta_{t}=\left(\beta_{0 t}, \beta_{1 t}, \ldots, \beta_{k t}\right)$ is a vector of time-dependent regression parameters, which evolve over time according to

$$
\beta_{j t}=\beta_{j, t-1}+\kappa_{j t} \eta_{j t}, \quad j=0, \ldots, k,
$$

where $\eta_{j t} \sim N\left(0, q_{j}^{2}\right)$ for $j=0, \ldots, k$, and $\kappa_{j t}$ is an unobserved uncorrelated $0 / 1$ process with $\operatorname{Pr}\left[\kappa_{j t}=1\right]=\pi_{j}$ for $j=0, \ldots, k$. Hence, the value of the $j$ th regression parameter $\beta_{j t}$ stays the same as $\beta_{j, t-1}$ unless $\kappa_{j t}=1$ in which case it changes with $\eta_{j t}$, see, for example, Koop and Potter (2004) and Giordani et al. (2006) for a similar approach. The predictor variables $x_{t}$ are demeaned to exclude that any break in one of the $\beta_{j t}$ implies also a break in the coefficient of the constant term, $\beta_{0 t}$. Then, $\beta_{0 t}$ represent the unconditional equity premium.

The specification in (4) implies that the regression parameters $\beta_{j t}, j=0, \ldots, k$, are allowed to change every time period, but they need not change at any point in time. The presence of a change is described by the latent binary random variable $\kappa_{j t}$, while the magnitude of the change is determined by $\eta_{j t}$, which is assumed to be normally distributed with mean zero. Note that the changes in the separate regression parameters are not restricted to coincide as in Pesaran and Timmermann (2002) but rather are allowed to occur at different points in time, see also Giordani et al. (2006).

While model uncertainty and structural breaks in the context of return prediction models have been studied in isolation, attempts to consider both features simultaneously are very rare, but see Pettenuzzo and Timmermann (2005). Using the representation of model uncertainty as given in (2), it actually turns out to be fairly straightforward to incorporate structural breaks as well, for example by adding the time-varying parameter specification as given in (4). Hence, we propose the following linear regression model for the excess stock return $r_{t}$ :

$$
r_{t}=\beta_{0 t}+\sum_{j=1}^{k} s_{j} \beta_{j t} x_{j t}+\varepsilon_{t},
$$

where $\varepsilon_{t} \sim N\left(0, \sigma^{2}\right)$ and $\beta_{t}=\left(\beta_{0 t}, \beta_{1 t}, \ldots, \beta_{k t}\right)^{\prime}$ evolves over time according to (4) as before.

For inference in our model (5) with (4) we opt for a Bayesian approach. This will provide the posterior distribution of the latent $\kappa_{j t}$ processes for $j=0, \ldots, k$ 
and $t=1, \ldots, T$. Bayesian inference on $S$ leads to posterior probabilities of the $2^{k}$ possible models which can in turn be used for Bayesian model selection and Bayesian model averaging. Notice that $\kappa_{j t}$ does not depend on $S$. At the same time the estimate of $\kappa_{j t}$ can be different across different values of $S$ and hence breaks can occur in different parameters and at different time periods across models. Below we first discuss prior specification, followed by a description of the posterior simulation algorithm.

\subsection{Prior Specification and Posterior Simulation}

The parameters in the model (5) with (4) are the variances of the residual returns, $\sigma^{2}$, and of the magnitude of the breaks in the regression parameters, $q_{0}^{2}, \ldots, q_{k}^{2}$, in addition to the variable inclusion probabilities $\lambda_{1}, \ldots, \lambda_{k}$ and the structural break probabilities $\pi_{0}, \ldots, \pi_{k}$. The model parameters are collected in the $(3(1+k) \times 1)$ vector $\theta=\left(\sigma^{2}, \lambda_{1}, \ldots, \lambda_{k}, q_{0}^{2}, \ldots, q_{k}^{2}, \pi_{0}, \ldots, \pi_{k}\right)$. To facilitate the posterior simulation we make use of independent conjugate priors. For the variance parameters we take the inverted Gamma-2 prior

$$
\sigma^{2} \sim \mathrm{IG}-2\left(\nu_{s}, \delta_{s}\right)
$$

and

$$
q_{j}^{2} \sim \mathrm{IG}-2\left(\nu_{j}, \delta_{j}\right)
$$

for $j=0, \ldots, k$, where the $\nu$ and $\delta$ are prior parameters which can be chosen to reflect the prior beliefs about the variances.

For the probability parameters we take Beta distributions,

$$
\begin{array}{ll}
\lambda_{j} \sim \operatorname{Beta}\left(a_{j}, b_{j}\right) & \text { for } j=1, \ldots, k, \text { and } \\
\pi_{j} \sim \operatorname{Beta}\left(c_{j}, d_{j}\right) & \text { for } j=0, \ldots, k .
\end{array}
$$

The parameters $a_{j}$ and $b_{j}$ can be set according to ones prior belief about the inclusion of the $j$ th explanatory variable in the model. Prior beliefs about structural breaks are incorporated through the parameters $c_{j}$ and $d_{j}$. Realistic values of these prior parameters depend on the problem at hand. In Section 3 we discuss the prior settings for our application.

Posterior results are obtained using the Gibbs sampler of Geman and Geman (1984) combined with the technique of data augmentation of Tanner and Wong (1987). The latent variables $S=\left(s_{1}, \ldots, s_{k}\right), B=\left\{\beta_{t}\right\}_{t=1}^{T}$ and $K=\left\{\kappa_{t}\right\}_{t=1}^{T}$ with $\kappa_{t}=\left(\kappa_{0 t}, \kappa_{1 t}, \ldots, \kappa_{k t}\right)$ are simulated alongside the model parameters $\theta$. 
The complete data likelihood function is given by

$$
\begin{aligned}
p(r, B, K, S \mid x, \theta)= & \prod_{t=1}^{T} p\left(r_{t} \mid S, x_{t}, \beta_{t}, \sigma^{2}\right) \prod_{t=1}^{T} p\left(\beta_{t} \mid \beta_{t-1}, \kappa_{t}, \theta\right) \\
& \prod_{j=1}^{k} \lambda_{j}^{s_{j}}\left(1-\lambda_{j}\right)^{1-s_{j}} \prod_{t=1}^{T} \prod_{j=0}^{k} \pi_{j}^{\kappa_{j t}}\left(1-\pi_{j}\right)^{1-\kappa_{j t}},
\end{aligned}
$$

where $r=\left(r_{1}, \ldots, r_{T}\right)$ and $x=\left(x_{1}^{\prime}, \ldots, x_{T}^{\prime}\right)^{\prime}$. The terms $p\left(r_{t} \mid S, x_{t}, \beta_{t}, \sigma^{2}\right)$ and $p\left(\beta_{t} \mid \beta_{t-1}, \kappa_{t}, \theta\right)$ are normal density functions, which follow directly from (5) and (4), respectively. If we combine (10) together with the prior density $p(\theta)$, which follows from (6)-(9), we obtain the posterior density

$$
p(\theta, B, K, S \mid r, x) \propto p(\theta) p(r, B, K, S \mid x, \theta) .
$$

To derive the Gibbs sampler we combine the Kuo and Mallick (1998) algorithm for variable selection and the efficient sampling algorithm of Gerlach et al. (2000) to handle the (occasional) structural breaks. The sampling scheme can be summarized as follows:

1. Draw $S$ conditional on $B, K, \theta, r$ and $x$.

2. Draw $K$ conditional on $S, \theta, r$ and $x$.

3. Draw $B$ conditional on $S, K, \theta, r$ and $x$.

4. Draw $\theta$ conditional $S, B, K, r$ and $x$.

Step 1 is done similarly to Kuo and Mallick (1998), which is a simplified version of the George and McCulloch (1993) algorithm. Starting from the previous iteration, the variable $S$ is drawn from its full conditional posterior distribution. The complete data likelihood function (10) is computed for $s_{j}=0$ and $s_{j}=1$ resulting in $p_{j, 0}$ and $p_{j, 1}$. The full conditional posterior is then given by

$$
\operatorname{Pr}\left[s_{j}=1 \mid r, x, \theta, B, K, S_{-j}\right]=\frac{p_{j, 1}}{p_{j, 0}+p_{j, 1}},
$$

for $j=1, \ldots, k$, where $S_{-j}=\left(s_{1}, \ldots, s_{j-1}, s_{j+1}, \ldots, s_{k}\right)$.

The (occasional) structural breaks, measured by the latent variable $\kappa_{j t}$, are drawn in step 2 using the algorithm of Gerlach et al. (2000), which derives its efficiency from generating $\kappa_{j t}$ without conditioning on the states $\beta_{j t}$. The conditional posterior density for $\kappa_{t}, t=1, \ldots, T$ unconditional on $B$ is

$$
\begin{aligned}
p\left(\kappa_{t} \mid K_{-t}, S, \theta, r, x\right) & \propto p(r \mid K, S, \theta, x) p\left(\kappa_{t} \mid K_{-t}, S, \theta, x\right) \\
\propto & p\left(r_{t+1}, \ldots, r_{T} \mid r_{1}, \ldots, r_{t}, S, \theta, x\right) \\
& \quad p\left(r_{t} \mid r_{1}, \ldots, r_{t-1}, \kappa_{1}, \ldots, \kappa_{t}, S, \theta, x\right) p\left(\kappa_{t} \mid K_{-t}, S, \theta, x\right),
\end{aligned}
$$


where $K_{-t}=\left\{\kappa_{s}\right\}_{s=1, s \neq t}^{T}$. Note that the term $p\left(\kappa_{t} \mid K_{-t}, S, \theta, x\right)$ is simply given by $\prod_{j=0}^{k} \pi_{j}^{\kappa_{j t}}\left(1-\pi_{j}\right)^{1-\kappa_{j t}}$ given that $\kappa_{j t}$ does not depend on $s_{j}$. The two remaining densities $p\left(r_{t+1}, \ldots, r_{T} \mid r_{1}, \ldots, r_{t}, S, \theta, x\right)$ and $p\left(r_{t} \mid r_{1}, \ldots, r_{t-1}, \kappa_{1}, \ldots, \kappa_{t}, S, \theta, x\right)$ can easily be evaluated as shown in Gerlach et al. (2000). Because $\kappa_{t}$ can take a finite number of values, the integrating constant can easily be computed by normalization.

The full conditional posterior density for the latent regression parameters $B$ in step 3 is computed using the simulation smoother as in Carter and Kohn (1994). The Kalman smoother is applied to derive the conditional mean and variance of the latent factors; for the initial value $\beta_{0}$ a multivariate normal prior with mean 0 is chosen.

Note in case the variable $x_{j}$ is not selected, the full conditional distributions of $\kappa_{j t}$ and $\beta_{j t}$ for $t=1, \ldots, T$ do not depend on the data $r$ and $x$. Hence, in this case we sample unconditionally from the process in (4) and the binary random process for $\kappa_{j t}$.

To sample the parameters $\theta$ in step 4 we can use standard results in Bayesian inference. Hence, the variance parameters $\sigma^{2}$ and $q_{j}^{2}$ are sampled from inverted Gamma-2 distributions and the probabilities $\pi_{j}$ and $\lambda_{j}$ are sampled from Beta distributions.

\subsection{Using the Posterior Results}

The output of the Gibbs sampler can be used to compute several quantities of interest. First, the marginal posterior distribution of the individual $s_{j}$ parameters $p\left(s_{j} \mid r, x\right)$ represents the posterior probability that variable $x_{j}$ is included in the model. This can used to assess the (relative) importance of the different predictor variables for forecasting stock returns. The interaction of different predictor variables can also be examined. For example, following Doppelhofer and Weeks (2005) the degree of dependence or jointness among two explanatory variables $x_{j}$ and $x_{l}$ can be formally computed by the following measure of jointness:

$$
J_{j, l}=\log \left(\frac{p\left(s_{j}=1 \cap s_{l}=1 \mid r, x\right)}{p\left(s_{j=1} \mid r, x\right) * p\left(s_{l}=1 \mid r, x\right)}\right)
$$

where the numerator is the posterior joint probability of inclusion of the couple of variables $x_{j}$ and $x_{l}$, and the denominator is the product of the marginal posterior probabilities of the inclusion of the $i$ th and $j$ th variables. We define two variables significant substitutes if $J_{j, l}<-1$, and significant complements if $J_{j, l}>1$. In addition, posterior model probabilities are easily obtained form the joint posterior of $S p(S \mid r, x)$. 
Second, we can use the simulated draws of $K$ to do inference on the occurrence of structural breaks in the regression parameters during the sample period. Obviously, one might consider the marginal posterior distribution of a single $\kappa_{j t} p\left(\kappa_{j t} \mid r, x\right)$, but the presence of contemporaneous breaks in different parameters can also be evaluated. Similarly, one can examine whether posterior evidence for breaks differs across models by conditioning on the inclusion/exclusion of certain variables in the model, for example, the posterior probability of a break in the regression parameter of variable $x_{j}$ given that variables $x_{l}$ and $x_{m}$ are included in the model is given by $p\left(\kappa_{j t} \mid s_{l}=s_{m}=1, r, x\right)$.

Third, the model in (5) with (4) can be used to predict future returns $r_{T+h}$ for $h \geq 1$. As our inference is Bayesian, we can explicitly take into account parameter uncertainty, uncertainty in variable selection, and uncertainty in the occurrence of structural breaks. In the empirical application in the next section, we focus on onestep ahead forecasting. For that reason the discussion below is limited to the case $h=1$, but it can be generalized to $h>1$ straightforwardly.

The one-step ahead predictive density of $r_{T+1}$ made at time $T$ conditional on $r$, $x$ and $x_{T+1}$ is given by

$$
\begin{aligned}
& p\left(r_{T+1} \mid r, x, x_{T+1}\right)=\iint \sum_{S} \sum_{K} \sum_{K_{T+1}} p\left(r_{T+1} \mid S, x_{T+1}, \beta_{T+1}, \sigma^{2}\right) \\
& p\left(\beta_{T+1} \mid \beta_{T}, \kappa_{T+1}, \theta\right) \prod_{j=0}^{k} \pi_{j}^{\kappa_{j, T+1}}\left(1-\pi_{j}\right)^{1-\kappa_{j, T+1}} p(B, K, S, \theta \mid r, x) d B d \theta
\end{aligned}
$$

where $p\left(r_{T+1} \mid S, x_{T+1}, \beta_{T+1}, \sigma^{2}\right)$ and $p\left(\beta_{T} \mid \beta_{T-1}, \kappa_{T+1}, \theta\right)$ follow directly from (5) and (4) and were $p(B, K, S, \theta \mid r, x)$ is the posterior density.

As we average over the posterior distribution of $S$ we implicitly take a weighted average over all possible model specifications, where the weights are the posterior model probabilities. The posterior distribution also reflects our posterior beliefs about the in-sample structural breaks $K$. Finally, note that we also average with respect the unknown $K_{T+1}$ variable to account for the possibility that a break may occur in the out-of-sample period $T+1$, where the weights are given by $\prod_{j=0}^{k} \pi_{j}^{\kappa_{j, T+1}}\left(1-\pi_{j}\right)^{1-\kappa_{j, T+1}}$.

Simulating $r_{T+1}$ from the one-step ahead distribution (15) is in fact rather straightforward. In each step of the Gibbs sampler, we use the simulated values of $\pi_{j}$ to draw the out-of-sample values of $\kappa_{j, T+1}$ for $j=0, \ldots, k$. Given the simulated values of $\kappa_{j, T+1}$ and given the Gibbs draws of $q_{j}^{2}$ and $\beta_{T}$ one can simulate $\beta_{T+1}$ using (4). Equation (5) in combination with the simulated value of $\beta_{T+1}$ and the current Gibbs draws of $S$ and $\sigma^{2}$ then provide a simulated value for $r_{T+1}$. 
Of course, often forecasting returns in itself is not the ultimate goal, but rather a means for determining the optimal asset allocation, for example. We postpone a detailed discussion of this issue in the context of our empirical application to Section 4 .

\section{Model uncertainty and structural breaks in re- turn forecasting models for the S\&P 500}

In the remainder of this paper we report results from an empirical application of the approach developed in Section 2 to predicting US stock returns using a set of 11 financial and macro-economic predictor variables. We start with a brief description of the data set and the choices made for prior specification. Next, we present full-sample estimation results, which can be considered as an ex-post analysis of the occurrence of structural breaks and the relevance of the different forecasting variables. An exante recursive forecasting experiment, which assesses the usefulness of our approach for predicting stock returns and active investment strategies in real-time is taken up in the following section.

\subsection{Data}

We use an update of the data set of Marquering and Verbeek (2004) covering the period from January 1966 until December 2005, for a total of 480 monthly observations. We use as our dependent variable the continuously compounded monthly return on the S\&P 500 index in excess of the 3-month T-Bill rate. The set of potential predictor variables includes the price-earnings ratio $(P E)$, the dividend yield $(D Y)$, the 3 -month T-Bill rate (I3), the 12-month Treasury Bond rate $(I 12)$, annual inflation $(I N F)$, the annual growth rate of industrial production $(I P)$, annual growth of the monetary base $(M B)$, the commercial paper-Treasury yield spread $(C P)$ and the logarithmic transformation of the realized monthly volatility $(\mathrm{Vol})$, which is computed as an adjusted estimator based upon the assumption that daily returns in month $t$ are appropriately described by a first-order autoregressive process, following French et al. (1987) and Akgiray (1989). To avoid look-ahead bias, the financial variables are included with a one-month lag and the macroeconomic variables with a two-month lag. The second lag of the short- and long-term interest rates are included as well to allow for the possibility that changes in the interest rates affect investment decisions more than their levels. 


\subsection{Prior specification}

For the hyperparameters $a_{j}$ and $b_{j}$ in the beta distribution that is used for the prior probability of inclusion of the variable $x_{j t}$ we assume $a_{j}=b_{j}=+\infty$, implying that $\operatorname{Pr}\left[s_{j}=1\right]=\lambda_{j}=0.5$ for all $j=1, \ldots, k$. As in our framework the $\lambda_{j}$ 's are independent across $j$, a 'diffuse' prior for $\lambda_{j}$ implies that all individual models have equal prior probability, as discussed in Fernández et al. (2001). For the hyperparameters $c_{j}$ and $d_{j}$ in the Beta distribution for the prior probability of breaks in the regression parameters, $p_{j}=\operatorname{Pr}\left[\kappa_{j t}=1\right]$, we assume $c_{j}=0.7$ and $d_{j}=35$ for any $j$. This implies that the prior mean duration between breaks in a particular regression parameter is equal to 51 months. Finally, for the conjugate inverted Gamma-2 densities for $\sigma^{2}$ and $\omega_{j}^{2}$, we assume a very peaked prior for the $\omega_{j}^{2}$ with mode near zero to limit the number of potential breaks.

\subsection{Full-sample estimation results}

We estimate the linear regression model with variable selection and occasional structural breaks in the parameters (5) with (4) using the complete sample period from January 1966 until December 2005. This enables us to provide an ex-post analysis of the relevance of the different predictor variables and possible breaks in their regression parameters. ${ }^{1}$

Table 1 provides the posterior mean for the probability of inclusion parameter $\lambda_{j}$. We observe that the first lag of the 3 -month T-Bill rate $I 3_{-1}$ is included with probability 1 . This perhaps is not surprising given that the dependent variable is the stock return in excess of the 3-month rate. The only other variables for which the posterior probability of inclusion is higher than the prior probability are the first lag of 12 -month T-bond rate $I 12_{-1}(0.775)$, the dividend yield $D Y_{-1}(0.727)$ and the price-earnings ratio $P E_{-1}(0.587)$. Obviously, bonds are alternative investments to stocks, in particular during bear markets, such that it is to be expected that they have some predictive power for stock returns. By contrast, the dividend yield is often referred to as an indicator of stock market performance. Note that the second lags of both interest rates have rather low posterior inclusion probabilities, indicating that movements in interest rates have substantially lower in-sample predictive power than the interest rate levels. The credit spread $C P_{-1}$ and stock

\footnotetext{
${ }^{1}$ We point out that the predictor variables are demeaned to exclude that possible breaks in the relation between the excess returns and some predictors imply also a breaks in the coefficient of the constant term.
} 
return volatility $\mathrm{LVOL}_{-1}$ have particular low posterior inclusion probabilities, indicating that these variables have not been useful as predictors of stock returns over the sample period considered. Finally, all three macroeconomic variables have posterior inclusion probabilities slightly below the prior value of 0.5 , suggesting lower predictive power than the financial variables.

Additional insight into the variable selection results can be obtained from the joint selection of different variables. For that purpose, Table 2 presents the posterior joint probabilities of inclusion for all possible pairs of variables. Note that the prior probability of joint inclusion for any two variables is equal to 0.25 , given that all individual prior probabilities are equal to 0.5 and independent across variables. Table 3 shows the values of the measure of jointness results of Doppelhofer and Weeks (2005). Note that the jointness measure involving the 3-month T-Bill rate takes the value zero by construction. Obviously, the results in Table 2 partly follow directly from the variable-specific selection probabilities in Table 1. For example, given that the dividend yield and the two interest rate variables have such high individual probabilities of inclusion, their combinations have high posterior probability to be selected together as well. The measure of jointness for $\left(D Y_{-1}, I 12_{-1}\right)$ has a correspondingly large value. The couple $\left(P E_{-1}, D Y_{-1}\right)$ has a posterior probability of being selected together of 0.316 , which is very close to the lower bound (0.314) that is possible given their individual inclusion probabilities. Although not borne out by their coefficient of jointness, this indicates that these variables are close substitutes. This is not surprising given that both the price-earnings ratio and the dividend yield are well-accepted valuation measures having similar predictive content for the development of the stock market. For the monetary base growth variable $M B_{-2}$ the posterior probability of joint inclusion with the dividend-yield, price-earnings ratio, and both short- and long-term interest rates is higher than its prior, while the corresponding jointness measures in Table 3 confirm that money growth is complementary to these financial variables. Almost all other combinations of variables have posterior probabilities lower than the prior value of 0.25 . Nevertheless, the jointness measures for industrial production growth $I P_{-2}$ and volatility $L V O L_{-1}$ indicate that these variables may include some useful information that complements the financial variables.

Finally, Table 4 provides the ten models which have the highest probabilities to be selected. The conclusions from this table agree with the findings from Tables 1-3 as discussed above. First, the variables $I 3_{-1}$ and $I 12_{-1}$ are always included in the most likely models. Second, either the dividend yield $D Y_{-1}$ or the price-earnings 
ratio $P E_{-1}$ also enters the model, but these two variables are not often included together because they are complements. Third, even though in several models one of the macro-economic variables is included, their importance does not seem very large. Finally, it is worth nothing that the sum of the posterior probabilities for these ten models is larger than 0.5, suggesting that financial variables are the most important predictor variables for stock returns.

We now turn to the analysis of the regression parameters and possible structural breaks therein. Figure 1 shows the posterior mean for the latent binary variable $\kappa_{j t}$ governing the occurrence of changes in the regression parameters, together with the associated posterior mean for $\beta_{j t}$. For the latter, 25th and 75th percentiles of the posterior distributions are also shown. One of the things that stands out most clearly from the graphs in Figure 1 is the spiky nature of the posterior mean of $\kappa_{j t}$, suggesting that the probabilities of structural breaks in the parameters vary considerably from one period to the next. ${ }^{2}$ This occurs for two reasons. First, $\kappa_{j, t}$ can be different across different values of $S$, such that breaks can occur at different times across models. Second, in case a break is estimated to have occurred in a certain month, the probability of a break in the next month will be much lower. Despite the volatile behavior of the break probabilities, three periods with considerable probability mass can be identified: during the years 1974-1975, around 1982 and around 2001. Political reasons and the oil price shocks provide possible explanations for the first break period. The change of the Federal Reserve's operating procedures at the beginning of the 1980's explain the second break, and the crash of the internet bubble the third one. Also note that the stock market crash in October 1987 gives rise to an isolated jump in the break probability for some variables (notably the constant $C$, the price-earnings ratio $P E_{-1}$, industrial production $I P_{-2}$, the credit spread $C P_{-1}$ and volatility $\left.L V O L_{-1}\right)$. This seems to suggest that this event did not give rise to a permanent change in the relationship between stock returns and the predictor variables, but rather is identified as an outlier.

The 25th-75th percentile bands for the regression parameters $\beta_{j t}$ in Figure 1 are quite wide and furthermore, the magnitudes of the changes in the posterior mean when a break occurs do not seem very large. This is due to the fact that variables are not always selected in the model, and when they are not, values for their regression parameters are drawn from their prior distributions. This explains, for example, why the variables with a low probability to be selected have rather flat posterior means

\footnotetext{
${ }^{2}$ Recall that the posterior mean of $\kappa_{j t}$ is identical to the posterior probability of a break occurring in the regression parameter for the $j$ th variable $x_{j t}$ at time $t$.
} 
for $\beta_{j, t}$, around the prior value. In Figure 2 we therefore consider the posterior densities for $\kappa_{j t}$ and $\beta_{j, t}$ conditional on inclusion of the variable $j$, that is $s_{j}=1$. Obviously, the posterior probabilities of structural breaks are much higher in this case, while the magnitudes of the breaks become larger and vary considerably over the set of variables. Several interesting findings emerge. First, the pattern of the intercept $\beta_{0 t}$ reveals a gradual increase in the unconditional equity premium during the 1980s and 1990s, followed by a decline just before the turn of the millennium. Second, for the price-earnings ratio $P E_{-1}$ and the dividend yield $D Y_{-1}$ the most substantial changes in parameters occur during the period 1999-2002 (in addition to the drop in the $P E_{-1}$ coefficient during the second half of the 1970s). These changes reflect the large decline in the dividend yield and corresponding large increase in the price-earnings ratio due to the dramatic boom of stock prices during that period. Third, the largest breaks in the coefficients related to interest rates appear to have occurred around 1982, around the time the Federal Reserve changed its monetary policy. Fourth, the coefficients related to inflation and industrial production growth display the largest change around 1974, due to the oil price shocks and the higher level of inflation and slowdown in economic growth that followed. Fifth and finally, the coefficients of the monetary base and the credit spread display very large breaks at October 1987. Hence, contrary to our prior observation that the stock market crash probably is considered to be an outlier, it does seem to have led to structural breaks in at least some relationships between stock returns and predictor variables. In that respect, the pattern in the coefficient of volatility also is interesting, showing a gradual decline up to the moment of the crash, and a gradual increase thereafter.

\section{Active investment strategies allowing for model uncertainty and structural breaks}

The full-sample results presented in the previous section provides a useful ex post characterization of the (relative) importance of financial and macroeconomic variables as predictors in return forecasting models and of possible breaks in the regression parameters. For an investor, both issues of variable selection and model instability are most interesting from an ex ante perspective. That is, the relevant questions are whether we can identify the appropriate predictor variables and detect structural breaks in regression parameters in real time, and how these may affect investment decisions. Answering these questions is the purpose of this section. 


\subsection{A utility-based performance measure}

Several papers consider the effects of either model uncertainty or model instability on optimal asset allocation decisions, see Kandel and Stambaugh (1996), Barberis (2000), Avramov (2002) and Pettenuzzo and Timmermann (2005). Most of these analyses focus on horizon effects, that is the issue how uncertainty about the relevant predictor variables or the possibility of structural breaks changes the decisions of investors with different horizons, typically ranging from a single month up to ten years. Here we only consider an active short-term investor, with an investment horizon of one month. The investor's portfolio consists of stocks and riskfree bonds only. At the start of each month $T+1$, the investor decides upon the fraction of her portfolio to be invested in stocks $w_{T+1}$, based upon a forecast of the excess stock return $r_{T+1}$. The investor is assumed to maximize a power utility function with coefficient of relative risk aversion $\gamma$ :

$$
u\left(W_{T+1}\right)=\frac{W_{T+1}^{1-\gamma}}{1-\gamma}, \quad \gamma>0,
$$

where $W_{T+1}$ is the wealth at the end of period $T+1$, which is equal to

$$
W_{T+1}=W_{T}\left(\left(1-w_{T+1}\right) \exp \left(r_{f, T+1}\right)+w_{T+1} \exp \left(r_{f, T+1}+r_{T+1}\right)\right),
$$

where $W_{T}$ denotes initial wealth, and where $r_{f, T+1}$ is the riskfree rate.

Without loss of generality we set initial wealth at one, $W_{T}=1$, such that the investor's optimization problem is given by

$\max _{w_{T+1}} E_{T}\left(u\left(W_{T+1}\right)\right)=\max _{w_{T+1}} E_{T}\left(\frac{\left(\left(1-w_{T+1}\right) \exp \left(r_{f, T+1}\right)+w_{T+1} \exp \left(r_{f, T+1}+r_{T+1}\right)\right)^{1-\gamma}}{1-\gamma}\right)$,

where $E_{T}$ is the conditional expectation given information at time $T$. How this expectation is computed depends on the treatment of model uncertainty and model instability by the investor. Consider the most general case, both allowing for uncertainty concerning which predictor variables to include and allowing for the possibility of structural breaks in the regressions parameters, as given by model (5) with (4). The marginal predictive density for future excess stock returns $p\left(r_{T+1} \mid r, x, x_{T+1}\right)$ in (15) should then be used to derive the proportion of the portfolio allocated to stocks according to (18). That is, the investor solves the following problem:

$$
\max _{w_{T+1}} \int u\left(W_{T+1}\right) p\left(r_{T+1} \mid r, x, x_{T+1}\right) d r_{T+1} .
$$

The integral in (19) is approximated by generating $G$ independent draws $\left\{r_{T+1}^{g}\right\}_{g=1}^{G}$ from the predictive density $p\left(r_{T+1} \mid r, x, x_{T+1}\right)$, and then using a constraint numerical 
optimization method to maximize the quantity:

$$
\frac{1}{G} \sum_{g=1}^{G}\left(\frac{\left(\left(1-w_{T+1}\right) \exp \left(r_{f, T+1}\right)+w_{T+1} \exp \left(r_{f, T+1}+r_{T+1}^{g}\right)\right)^{1-\gamma}}{1-\gamma}\right)
$$

Two further cases are included in the empirical analysis below. First, we consider an investor who incorporates model uncertainty but ignores the possibility of structural breaks in the regression parameters. This investor obtains a forecast of the excess stock return $r_{T+1}$ from model (5) but with $\beta_{j t}=\beta_{j}$ for $j=0,1, \ldots, k$ and $t=1, \ldots, T+1$. Second, we consider an investor who also is ignorant about model uncertainty and simply includes all available predictor variables in the model, effectively using the benchmark model (1) for return forecasting.

As explained by Barberis (2000), the weight $w_{T+1}$ in (17) cannot be left unconstrained in the optimization problem (18) as expected utility would be equal to $-\infty$ in that case. We consider the following two restrictions on $w_{T+1}$. First, we restrict $w_{T+1} \in[-1,2]$, allowing some extent of short-sales and leveraging of the portfolio. Second, we do not allow for short-sales or leveraging at all, by constraining $w_{T+1}$ to be in the $[0,1]$ interval. Hence, in total we consider six active investment strategies. For comparison, we include three static benchmark strategies: I) holding stocks only, II) holding a portfolio consisting of $50 \%$ stocks and $50 \%$ bonds, and III) holding bonds only.

We evaluate the different investment strategies by computing the ex post utility levels substituting the realized return of the portfolios at time $T+1$ in (18). Total utility is then obtained as the sum of $u\left(W_{T+1}\right)$ across all investment periods $T=$ $T_{0}, \ldots, T_{0}+T^{*}$. In order to compare two alternative strategies we compute the return that equates their average utilities. For example, suppose we compare the strategy based on excess return forecasts from the benchmark model (1) with a fixed set of predictor variables and constant regression parameters to the strategy based on the general model (5) with (4) that incorporates model uncertainty and structural breaks. The wealth provided at time $T+1$ by the two resulting portfolios is denoted as $W_{1, T+1}$ and $W_{2, T+1}$, respectively. We then determine the value of $\Delta$ such that

$$
\sum_{T=T_{0}}^{T_{0}+T^{*}} u\left(W_{1, T+1}\right)=\sum_{T=T_{0}}^{T_{0}+T^{*}} u\left(W_{2, T+1} / \exp (\Delta)\right)
$$

Following Fleming et al. (2001), we interpret $\Delta$ as the maximum performance fee the investor would be willing to pay to switch from the first strategy to the second. In that sense, $\Delta$ represents the economic value of model uncertainty and model instability, in the example above. 
Finally, the portfolio weights in the active investment strategies change every month, and the portfolio must be rebalanced accordingly. Hence, transaction costs play a non-trivial role and should be taken into account in evaluating the relative performance of different strategies. Rebalancing the portfolio at the start of month $T+1$ means that the weight invested in stocks is changed from $w_{T}$ to $w_{T+1}$. We assume that transaction costs amount to a fixed percentage $c$ on each traded dollar. Setting the initial wealth $W_{T}$ equal to 1 for simplicity, transaction costs at time $T+1$ are equal to

$$
c_{T+1}=2 c\left|w_{T+1}-w_{T}\right|
$$

where the multiplication by 2 follows from the fact that the investor rebalances her investments in both stocks and bonds. The net portfolio return is then given by $r_{T+1}-c_{T+1}$. We apply two scenarios with transaction costs of $0.1 \%$ and $0.5 \%$. Note that for a passive strategy the inclusion of transaction costs matters only in buying the portfolio at the beginning.

\subsection{Empirical Results}

The analysis for the active investment strategies is implemented for the period from January 1976 until December 2005, involving $T^{*}=360$ one month ahead return forecasts. The models are estimated recursively using an expanding window of observations, with the first $T=120$ months being used to estimate the initial models that are used to obtain the first return prediction. The investment strategies are implemented for two levels of relative risk aversion, $\gamma=5$ and 10. Before we analyze the performance of the different portfolios, we summarize the statistical accuracy of the forecasts of the excess stock returns.

The forecasts obtained from the model allowing for uncertainty concerning which predictor variables to include and allowing for the possibility of structural breaks in the regressions parameters (5) with (4) have mean error (ME) of $0.33 \%$ and a root mean square prediction error (RMSPE) of $4.58 \%$. This is slightly more accurate than the linear and BMA forecasting models, both of which have RMSPEs equal to $4.64 \%$. The ME of these models are $0.45 \%$ and $0.41 \%$, respectively. Figure 3 shows five-year moving averages of the excess returns' RMSPE and the hit ratio, defined as the proportion of correctly predicted signs. Both graphs show that the model performs quite well until October 1987. The stock market crash causes a large upward jump in the RMSPE, and marks the beginning of a period with less accurate forecasts and a steady decline in the hit ratio. Forecast accuracy improves again considerably during the period 1991-1997 with the RMSPE reaching a low of 
just under $3 \%$ and the hit ratio peaking at 0.67 . Predictability of the stock returns then deteriorates dramatically due to the internet bubble and its burst, and the hit ratio sharply drops to less than 0.4 in 2004. In sum, predictive accuracy varies considerably over time, even if a flexible forecast approach allowing for structural breaks and model uncertainty is employed.

Table 5 provides summary statistics for the performance of the nine different investment strategies considered, ignoring transaction costs for the moment. In addition to the total utility levels and the performance fee $\Delta$ relative to the buyand-hold stock portfolio, we report traditional performance measures including the annualized mean and standard deviation of portfolio returns, and the Sharpe ratio (computed as the ratio of the mean monthly excess return on the portfolio and the monthly standard deviation of the portfolio return).

Over the complete investment period from January 1976 until December 2005, the average annualized return on the buy-and-hold stock portfolio is $11.91 \%$ with an estimated unconditional standard deviation of $15 \%$, while the bonds portfolio provides a mean return of $5.82 \%$ with a standard deviation of $0.85 \%$. The Sharpe ratio of the stock portfolio is 0.117 , while for the bond portfolio it is zero by construction. In terms of utility levels, the buy-and-hold mixed portfolio consisting of $50 \%$ stocks and $50 \%$ bonds renders the best results.

Next consider the active investment strategies based on excess return forecasts that account for model uncertainty and breaks (Strategies IV and VII). We observe that these all render lower average returns than the buy-and-hold stock portfolio. At the same time, portfolio risk is reduced considerably as well. For example, the restricted portfolios render return standard deviations that are $8.0 \%$ and $6.1 \%$ for $\gamma=5$ and 10, respectively, compared to $15 \%$ for the passive stock portfolio. This reduction in volatility comes at the cost of lower mean returns by $2.2 \%$ and $3.2 \%$ for low and high risk averse investors, respectively. Despite this substantial return sacrifice, the Sharpe ratios of the active portfolios are higher at 0.138 and 0.133 . The benefits of the active investment strategy also are revealed clearly by the performance fee $\Delta$. We find that the investor would be willing to pay 130 and 700 basis points to switch from the passive to the active strategy. The passive mixed portfolio is outperformed as well, although the estimates of $\Delta$ are considerably lower at 45 and 64 basis points. The reduction in average returns is less for the unrestricted portfolios, but the corresponding reduction of return volatility also is much smaller such that the resulting Sharpe ratios are below that of the passive stock portfolio. For the investor with low risk aversion $(\gamma=5)$, the passive strategy now renders 
higher utility, resulting in a negative performance fee of -140 basispoints for the active strategy. The high risk averse still prefers the active strategy though, and would be willing to pay 475 basis points annually to trade it against the buy-andhold stock portfolio.

The performance of the other active investment strategies based on excess return forecasts from more restricted models is less convincing. Although these strategies lower the volatility of portfolio returns even more than the strategy based on forecasts from the general model, the reduction in average returns is considerably larger as well. For example, the restricted portfolio based on excess return forecasts from the model that only accounts for model uncertainty but ignores structural breaks (Strategy VIII) renders volatility of $1.1 \%$ but at a mean return of only $6.1 \%$ for $\gamma=10$. In all cases the resulting Sharpe ratios is lower compared to the portfolios based on the general model. Also in terms of the utility levels and performance fee, Strategy VII achieves the best performance.

Figure 3 suggests that the accuracy of the excess return forecasts varies considerably over time. How this affects the performance of the active strategies can be seen from Table 6, which shows performance statistics for three sub-periods each covering a decade for the investor with high relative risk aversion $(\gamma=10) .{ }^{3}$ We focus on the restricted active portfolios that results from forecasts of the general model allowing for model uncertainty and structural breaks (Strategy VII). Its performance is quite impressive during the first decade of the investment period, from January 1976 until December 1985, with a Sharpe ratio of 0.207, more than double the Sharpe ratios of the passive portfolios held in Strategies I and II. This is due to the fact that the mean return of the active strategy during this period is actually higher than the mean return of the buy-and-hold portfolio (14.4\% compared to $13.2 \%$ ), while volatility is reduced by about $40 \%$. The corresponding performance fees are positive and large. The Sharpe ratios of the active and passive strategies are exactly equal during the second decade from January 1986 until December 1995, although in terms of utility level the buy-and-hold stock portfolio is still outperformed by the active strategy. The mixed portfolio achieves exactly the same level of utility however, resulting in a performance fee close to zero. The active strategy's performance deteriorates during the third and final decade, from January 1996 until December 2005. Although the reduction in portfolio returns' volatility is of the same magnitude as before, the loss in average return is much larger. This results in a Sharpe ratio of

\footnotetext{
${ }^{3}$ Sub-sample results for the investor with low relative risk aversion $(\gamma=5)$ are qualitatively similar and therefore not shown to save space. Detailed results are available upon request.
} 
0.019, compared to 0.094 for the passive portfolios. It is quite remarkable then that the active strategy still achieved higher utility than the buy-and-hold stock portfolio (-13.06 compared to -13.68). The mixed passive portfolio in turn renders higher utility than the active strategy, resulting in a negative performance fee. In sum, it seems that the performance of the active strategies has gradually declined over time.

Our analysis of the active investment strategies so far has ignored transaction costs. Obviously, their effects on the strategies; performance crucially depends on the average absolute change in portfolio weights, see (22). Figure 4 shows the portfolio weight for stocks in the restricted portfolios based on excess stock return forecasts from the general model, allowing for model uncertainty and structural breaks in the regression parameters (Strategy VII). Although there are extended periods of time when the investment in stocks is at high or low levels, month-to-month variation in the portfolio weight seems quite substantial. Hence, a proper analysis of the effects of transaction costs is warranted.

Tables 8 and 8 present results for the complete 30-year investment period for low $(0.1 \%)$ and moderate $(0.5 \%)$ levels of transaction costs, respectively. The presence of transaction costs obviously hurts the active strategies' performance. For low transaction cost levels, the restricted portfolio based on return forecasts from the general model continues to outperform the buy-and-hold stock and mixed portfolios, although the performance fees $\Delta$ become somewhat lower. For moderate transaction cost levels, only the high risk averse investor prefers the active strategy over the passive stock portfolio. For both levels of risk aversion considered, the mixed portfolio renders superior results on all measures considered: it has higher mean return, lower volatility (and thus a higher Sharpe ratio) and higher utility.

\section{Conclusion}

Optimal portfolio decisions force investors to make a number of important decisions concerning the return forecasting model used. These decisions involve in particular the treatment of different sources of uncertainty, about the relevant predictor variables (model uncertainty), the values of the regression parameters (parameter uncertainty), and their stability (structural breaks). In this paper we have developed a framework to incorporate all three sources of uncertainty simultaneously. This extends previous research allowing for either parameter uncertainty and model uncertainty (Avramov (2002), Cremers (2002)), or parameter uncertainty and parameter instability (Pesaran et al. (2004)). 
Our empirical results suggest, first, that over the period 1966-2005, several structural breaks occurred in the relationship between US stock returns and predictor variables such as the dividend yield and interest rates. These changes appear to be caused by important events such as the oil crisis, changes in monetary policy, and the October 1987 stock market crash. Second, we find that allowing for model uncertainty, and structural breaks has considerable economic value. A typical investor would be willing to pay up to several hundreds of basis points annually to switch from a passive buy-and-hold strategy to an active strategy based on a return forecasting model that allows for model and parameter uncertainty as well as structural breaks in the regression parameters. The active strategy that incorporates all three sources of uncertainty performs considerably better than strategies based on more restricted return forecasting models. 


\section{References}

Akgiray, V. (1989), Conditional Heteroskedasticity in Time Series of Stock Returns: Evidences and Forecasts, Journal of Business, 62, 55-80.

Avramov, D. (2002), Stock Return Predictability and Model Uncertainty, Journal of Financial Economics, 64, 423-458.

Barberis, N. (2000), Investing for the Long Run When Returns Are Predictable, Journal of Finance, 55, 225-264.

Carter, C. and R. Kohn (1994), On Gibbs Sampling for State-Space Models, Biometrika, 81, 541-553.

Cremers, K. (2002), Stock Return Predictability: A Bayesian Model Selection Perspective, Review of Financial Studies, 15, 1223-1249.

Doppelhofer, G. and M. Weeks (2005), Jointness of Growth Determinants, Working paper.

Fernández, C., E. Ley, and M. Steel (2001), Benchmark priors for Bayesian model averaging, Journal of Econometrics, 381-427.

Fleming, J., C. Kirby, and B. Ostdiek (2001), The Economic Value of Volatility Timing, Journal of Finance, 56, 329-352.

French, K., G. Schwert, and R. Stambaugh (1987), Expcted Stock Returns and Volatility, Journal of Financial Economics, 19, 3-29.

Geman, S. and D. Geman (1984), Stochastic Relaxation, Gibbs Distributions and the Bayesian Restoration of Images, IEEE Transaction on Pattern Analysis and Machine Intelligence, 6, 721-741.

George, E. I. and R. E. McCulloch (1993), Variable Selection Via Gibbs Sampling, Journal of the American Statistical Association, 88, 881-889.

Gerlach, R., C. Carter, and R. Kohn (2000), Efficient Bayesian Inference for Dynamic Mixture Models, Journal of the American Statistical Association, 95, 819-828.

Giordani, P., R. Kohn, and D. van Dijk (2006), A Unified Approach to Nonlinearity, Outliers and Structural Breaks, Journal of Econometrics, to appear.

Kandel, S. and R. Stambaugh (1996), On the Predictability of Stock Returns: An Asset Allocation Perspective, Journal of Finance, 51, 385-424.

Koop, G. and S. Potter (2004), Forecasting and Estimating Multiple Change-point Models with an Unknown Number of Change-points, Working paper.

Kuo, L. and B. Mallick (1998), Variable Selection for Regression Models, The Indian Journal of Statistics, 60, 65-81.

Marquering, W. and M. Verbeek (2004), The Economic Value of Predicting Stock Index Returns and Volatility, Journal of Financial and Quantitative Analysis, 39 (2), 407429 . 
Pesaran, M., D. Pettenuzzo, and A. Timmermann (2004), Forecasting Time Series Subject to Multiple Structural Breaks, Working paper.

Pesaran, M. and A. Timmermann (2002), Market Timing and Return Predictability Under Model Instability, Journal of Empirical Finance, 9, 495-510.

Pettenuzzo, D. and A. Timmermann (2005), Predictability of Stock Returns and Asset Allocation under Structural Breaks, Working paper.

Tanner, M. and W. Wong (1987), The Calculation of Posterior Distributions by Data Augmentation, Journal of the American Statistical Association, 82, 528-550. 
Table 1: Probability of variable selection

\begin{tabular}{lc}
\hline \hline Variable & $\begin{array}{c}\text { Mean posterior } \\
\text { inclusion probability }\end{array}$ \\
\hline$P E_{-1}$ & 0.587 \\
$D Y_{-1}$ & 0.727 \\
$I 3_{-1}$ & 1.000 \\
$I 3_{-2}$ & 0.169 \\
$I 12_{-1}$ & 0.775 \\
$I 12_{-2}$ & 0.095 \\
$I N F_{-2}$ & 0.208 \\
$I P_{-2}$ & 0.352 \\
$M B_{-2}$ & 0.435 \\
$C P_{-1}$ & 0.070 \\
$L V O L_{-1}$ & 0.094 \\
\hline
\end{tabular}

The table presents the marginal posterior probability of any single explanatory variable to be selected. 


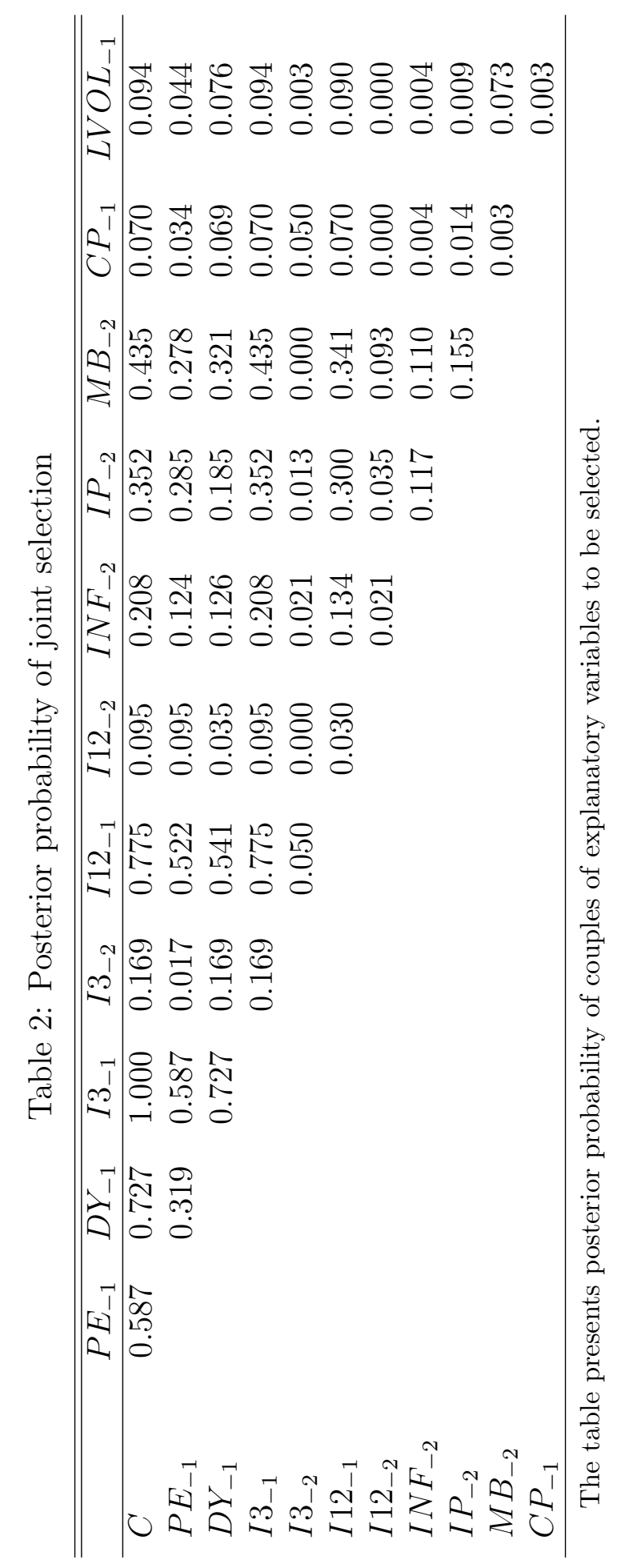




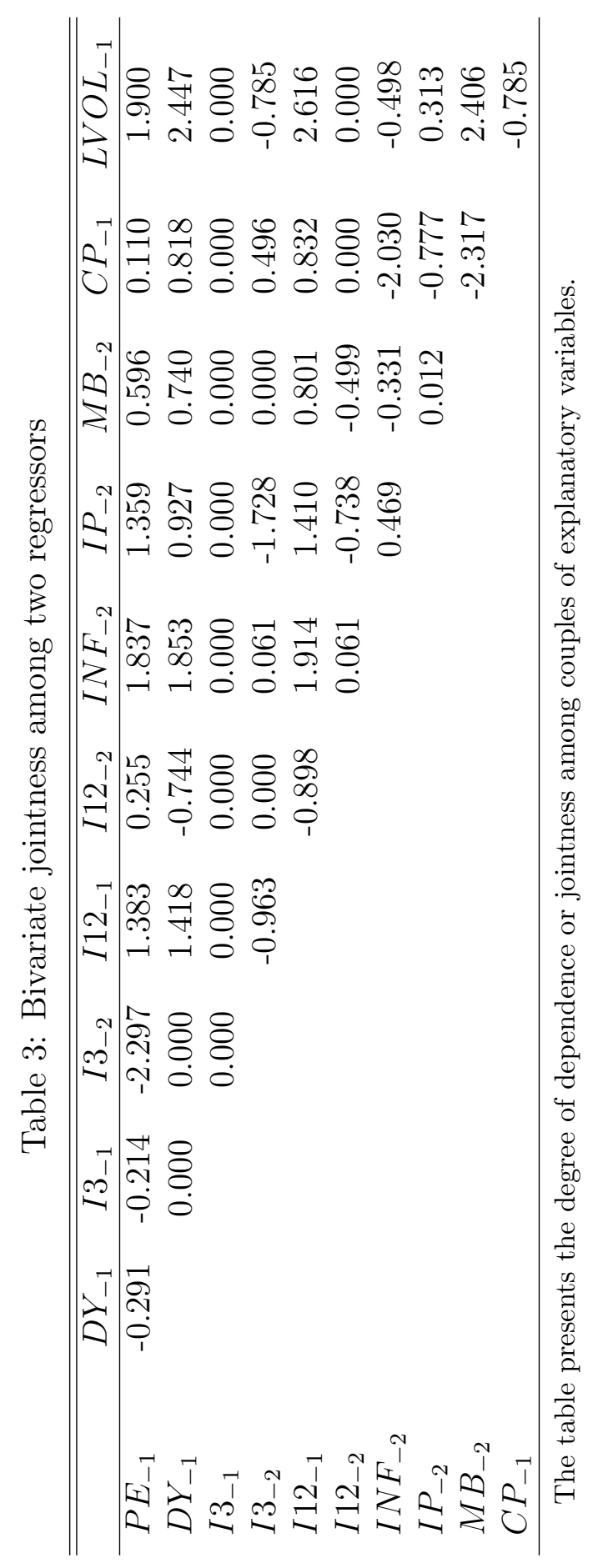




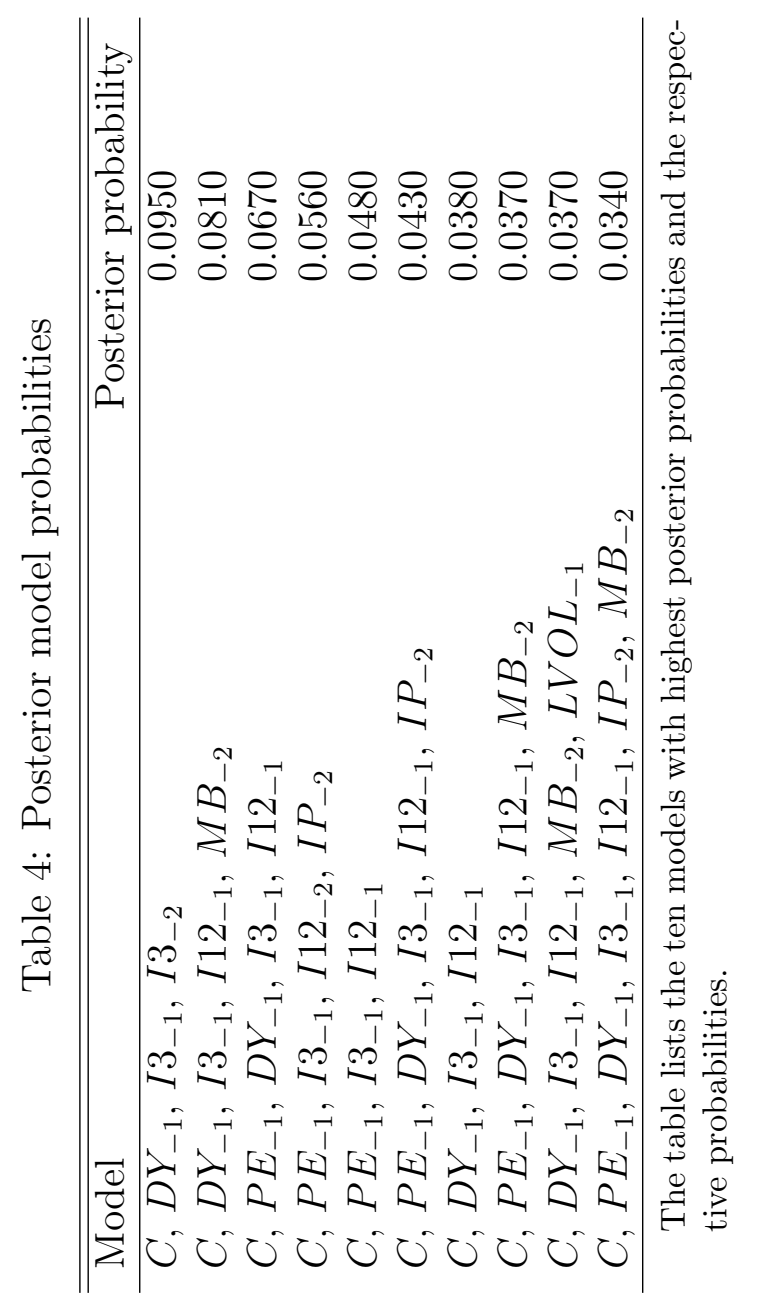


Table 5: No transaction costs

\begin{tabular}{lccccccc}
\hline \hline Strategy & Mean & St dev & SR & Utility & $\Delta_{m}$ & $\Delta_{0.5 m}$ & $\Delta_{b}$ \\
\cline { 1 - 1 } I: $100 \%$ market & & & Panel B: & $\gamma=5$ & & & \\
II: 50\% market & 0.119 & 0.150 & 0.117 & -87.88 & & & \\
III: 0\% market & 0.089 & 0.075 & 0.117 & -87.63 & & & \\
IV: BMASB & 0.058 & 0.008 & 0.000 & -88.28 & & & \\
V: BMA & 0.065 & 0.144 & 0.094 & -88.29 & -140.0 & -225.3 & -4.877 \\
VI: Linear & 0.081 & 0.067 & 0.109 & -88.07 & -64.19 & -149.5 & 70.88 \\
VII: BMASB (0,1) & 0.097 & 0.080 & 0.138 & -87.50 & 129.9 & 44.58 & 265.0 \\
VIII: BMA (0,1) & 0.065 & 0.017 & 0.109 & -88.07 & -64.19 & -149.5 & 70.88 \\
IV: Linear (0,1) & 0.077 & 0.050 & 0.109 & -87.83 & 16.61 & -68.75 & 151.7 \\
& & & & & & & \\
I: 100\% market & 0.119 & 0.150 & 0.117 & -39.96 & & & \\
II: 50\% market & 0.089 & 0.075 & 0.117 & -38.10 & & & \\
III: 0\% market & 0.058 & 0.008 & 0.000 & -38.30 & & & \\
IV: BMASB & 0.093 & 0.103 & 0.097 & -38.56 & 474.8 & -163.3 & -91.25 \\
V: BMA & 0.055 & 0.106 & -0.009 & -45.52 & -1736 & -2374 & -2302 \\
VI: Linear & 0.072 & 0.039 & 0.102 & -38.07 & 647.3 & 9.186 & 81.27 \\
VII: BMASB (0,1) & 0.087 & 0.061 & 0.133 & -37.91 & 701.8 & 63.66 & 135.7 \\
VIII: BMA (0,1) & 0.061 & 0.011 & 0.084 & -38.20 & 601.3 & -36.82 & 35.26 \\
IV: Linear (0,1) & 0.069 & 0.029 & 0.111 & -38.05 & 652.7 & 14.63 & 86.71 \\
\hline
\end{tabular}

The table presents the annualized average $\%$ return, the annualized standard deviation, the Sharpe ratio (SR), and the utility value of the 9 different strategies for the full forecasting sample period 1976:1-2005:12. The last 3 columns present the annualized return in basis points that an active strategy gives in surplus of the return of a passive strategy. 
Table 6: Subperiods, $\gamma=10$

\begin{tabular}{|c|c|c|c|c|c|c|c|}
\hline Strategy & Mean & St dev & $\overline{\mathrm{SR}}$ & Utility & $\Delta_{m}$ & $\Delta_{0.5 m}$ & $\Delta_{b}$ \\
\hline & & Panel A: & 1976:1 - & $1985: 12$ & & & \\
\hline I: $100 \%$ market & 0.133 & 0.140 & 0.097 & -12.88 & & & \\
\hline II: $50 \%$ market & 0.109 & 0.070 & 0.097 & -12.46 & & & \\
\hline III: $0 \%$ market & 0.086 & 0.008 & 0.000 & -12.51 & & & \\
\hline IV: BMASB & 0.197 & 0.148 & 0.217 & -12.26 & 665.1 & 223.2 & 270.9 \\
\hline $\mathrm{V}: \mathrm{BMA}$ & 0.097 & 0.121 & 0.028 & -13.11 & -230.7 & -672.6 & -624.9 \\
\hline VI: Linear & 0.132 & 0.055 & 0.240 & -12.19 & 734.0 & 292.2 & 339.9 \\
\hline VII: BMASB $(0,1)$ & 0.144 & 0.081 & 0.207 & -12.20 & 723.2 & 281.4 & 329.0 \\
\hline VIII: BMA $(0,1)$ & 0.088 & 0.009 & 0.086 & -12.48 & 425.0 & -16.79 & 30.90 \\
\hline IV: Linear $(0,1)$ & 0.114 & 0.036 & 0.229 & -12.28 & 639.5 & 197.7 & 245.3 \\
\hline & & Panel B: & 1986:1 - & 1995:12 & & & \\
\hline I: $100 \%$ market & 0.138 & 0.153 & 0.158 & -13.40 & & & \\
\hline II: $50 \%$ market & 0.096 & 0.077 & 0.158 & -12.65 & & & \\
\hline III: $0 \%$ market & 0.054 & 0.005 & 0.000 & -12.81 & & & \\
\hline IV: BMASB & 0.052 & 0.071 & -0.010 & -13.09 & 307.5 & -458.9 & -293.2 \\
\hline $\mathrm{V}: \mathrm{BMA}$ & 0.029 & 0.137 & -0.053 & -19.46 & -4976 & -5742 & -5576 \\
\hline VI: Linear & 0.057 & 0.025 & 0.041 & -12.79 & 614.2 & -152.2 & 13.48 \\
\hline VII: $\operatorname{BMASB}(0,1)$ & 0.078 & 0.044 & 0.158 & -12.65 & 764.6 & -1.783 & 163.9 \\
\hline VIII: BMA $(0,1)$ & 0.058 & 0.010 & 0.115 & -12.77 & 641.7 & -124.7 & 40.99 \\
\hline IV: Linear $(0,1)$ & 0.062 & 0.022 & 0.107 & -12.74 & 670.4 & -96.00 & 69.66 \\
\hline & & Panel C: & 1996:1 - & $2005: 12$ & & & \\
\hline I: $100 \%$ market & 0.086 & 0.157 & 0.094 & -13.68 & & & \\
\hline II: $50 \%$ market & 0.061 & 0.079 & 0.094 & -12.99 & & & \\
\hline III: $0 \%$ market & 0.035 & 0.005 & 0.000 & -12.99 & & & \\
\hline IV: BMASB & 0.031 & 0.063 & -0.019 & -13.22 & 461.7 & -237.7 & -233.9 \\
\hline $\mathrm{V}: \mathrm{BMA}$ & 0.038 & 0.009 & 0.095 & -12.96 & 729.4 & 29.98 & 33.81 \\
\hline VI: Linear & 0.027 & 0.022 & -0.102 & -13.08 & 598.5 & -101.0 & -97.14 \\
\hline VII: BMASB $(0,1)$ & 0.038 & 0.049 & 0.019 & -13.06 & 620.5 & -78.91 & -75.08 \\
\hline VIII: BMA $(0,1)$ & 0.038 & 0.009 & 0.095 & -12.96 & 729.4 & 29.99 & 33.82 \\
\hline IV: Linear $(0,1)$ & 0.031 & 0.020 & -0.053 & -13.04 & 648.0 & -51.46 & -47.63 \\
\hline
\end{tabular}

The table presents the annualized average \% return, the annualized standard deviation, the Sharpe ratio (SR), and the utility value of the 9 strategies for the full forecasting sample period 1986:11995:12. The last 3 columns present the annualized return in basis points that an active strategy gives in surplus of the return of a passive strategy. 
Table 7: $0.1 \%$ transaction costs

\begin{tabular}{lccccccc}
\hline \hline Strategy & Mean & St dev & SR & Utility & $\Delta_{m}$ & $\Delta_{0.5 m}$ & $\Delta_{b}$ \\
\cline { 1 - 3 } I: 100\% market & 0.119 & 0.150 & 0.117 & -87.88 & & & \\
II: 50\% market & 0.089 & 0.075 & 0.117 & -87.63 & & & \\
III: 0\% market & 0.058 & 0.008 & 0.000 & -88.28 & & & \\
IV: BMASB & 0.090 & 0.144 & 0.065 & -88.51 & -213.0 & -298.4 & -77.79 \\
V: BMA & 0.046 & 0.017 & -0.211 & -88.08 & -67.34 & -152.8 & 67.85 \\
VI: Linear & 0.064 & 0.067 & 0.026 & -87.93 & -18.003 & -103.43 & 117.2 \\
VII: BMASB (0,1) & 0.090 & 0.080 & 0.114 & -87.60 & 96.20 & 10.77 & 231.4 \\
VIII: BMA (0,1) & 0.064 & 0.017 & 0.098 & -88.08 & -67.34 & -152.8 & 67.85 \\
IV: Linear (0,1) & 0.074 & 0.049 & 0.092 & -87.87 & 1.862 & -83.57 & 137.1 \\
& & & & & & & \\
& & & Panel B: & $\gamma=10$ & & & \\
I: 100\% market & 0.119 & 0.150 & 0.117 & -39.96 & & & \\
II: 50\% market & 0.089 & 0.075 & 0.117 & -38.10 & & & \\
III: 0\% market & 0.058 & 0.008 & 0.000 & -38.30 & & & \\
IV: BMASB & 0.084 & 0.103 & 0.072 & -38.70 & 429.3 & -208.9 & -136.6 \\
V: BMA & 0.048 & 0.106 & -0.029 & -45.76 & -1807 & -2445 & -2373 \\
VI: Linear & 0.070 & 0.039 & 0.084 & -38.10 & 635.0 & -3.164 & 69.15 \\
VII: BMASB (0,1) & 0.082 & 0.061 & 0.111 & -37.98 & 678.1 & 40.02 & 112.3 \\
VIII: BMA (0,1) & 0.061 & 0.011 & 0.074 & -38.12 & 599.8 & -38.37 & 33.94 \\
IV: Linear (0,1) & 0.068 & 0.029 & 0.095 & -38.08 & 645.0 & 6.91 & 79.22 \\
\hline
\end{tabular}

The table presents the annualized average \% return, the annualized standard deviation, the Sharpe ratio (SR), and the utility value of the 9 different strategies for the full forecasting sample period 1976:1-2005:12 with transaction cost of 0.1\%. The last 3 columns present the annualized return in basis points that an active strategy gives in surplus of the return of a passive strategy. 
Table 8: $0.5 \%$ transaction costs

\begin{tabular}{lccccccc}
\hline \hline Strategy & Mean & St dev & SR & Utility & $\Delta_{m}$ & $\Delta_{0.5 m}$ & $\Delta_{b}$ \\
\cline { 1 - 1 } I: 100\% market & 0.119 & 0.150 & 0.117 & -87.88 & & & \\
II: 50\% market & 0.088 & 0.075 & 0.116 & -87.63 & & & \\
III: 0\% market & 0.058 & 0.009 & 0.000 & -88.28 & & & \\
IV: BMASB & 0.032 & 0.144 & -0.053 & -89.38 & -507.1 & -592.8 & -371.4 \\
V: BMA & -0.031 & 0.024 & -1.078 & -88.12 & -79.96 & -165.7 & 55.71 \\
VI: Linear & -0.003 & 0.068 & -0.262 & -88.22 & -114.22 & -200.0 & 21.45 \\
VII: BMASB (0,1) & 0.063 & 0.081 & 0.017 & -88.00 & -39.29 & -125.0 & 96.38 \\
VIII: BMA (0,1) & 0.061 & 0.017 & 0.051 & -88.12 & -79.96 & -165.7 & 55.71 \\
IV: Linear (0,1) & 0.062 & 0.049 & 0.022 & -88.05 & -57.21 & -142.9 & 78.47 \\
& & & & & & & \\
& & & Panel B: & $\gamma=10$ & & & \\
I: 100\% market & 0.119 & 0.150 & 0.117 & -39.96 & & & \\
II: 50\% market & 0.088 & 0.075 & 0.116 & -38.10 & & & \\
III: 0\% market & 0.058 & 0.009 & 0.000 & -38.31 & & & \\
IV: BMASB & 0.046 & 0.103 & -0.033 & -39.24 & 245.4 & -392.8 & -319.5 \\
V: BMA & 0.019 & 0.107 & -0.104 & -46.78 & -2101 & -2739 & -2666 \\
VI: Linear & 0.059 & 0.038 & 0.007 & -38.25 & 585.4 & -52.73 & 20.52 \\
VII: BMASB (0,1) & 0.063 & 0.062 & 0.022 & -38.25 & 583.0 & -55.19 & 18.07 \\
VIII: BMA (0,1) & 0.060 & 0.011 & 0.037 & -38.16 & 593.6 & -44.60 & 28.66 \\
IV: Linear (0,1) & 0.061 & 0.028 & 0.031 & -38.17 & 614.1 & -24.03 & 49.23 \\
\hline
\end{tabular}

The table presents the annualized average $\%$ return, the annualized standard deviation, the Sharpe ratio (SR), and the utility value of the 9 different strategies for the full forecasting sample period 1976:1-2005:12 with transaction cost of 0.5\%. The last 3 columns present the annualized return in basis points that an active strategy gives in surplus of the return of a passive strategy. 
Figure 1: Marginal posterior densities of the breaks and $\beta$ parameters
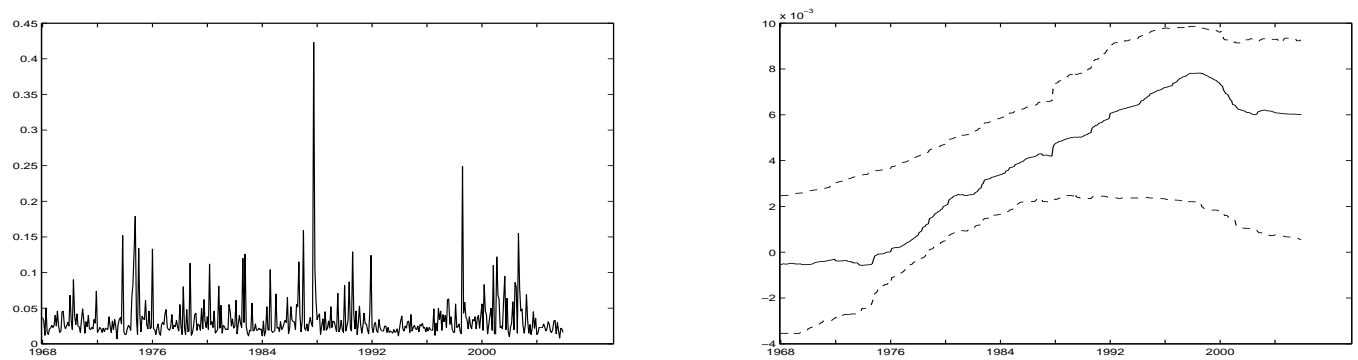

(a) Constant
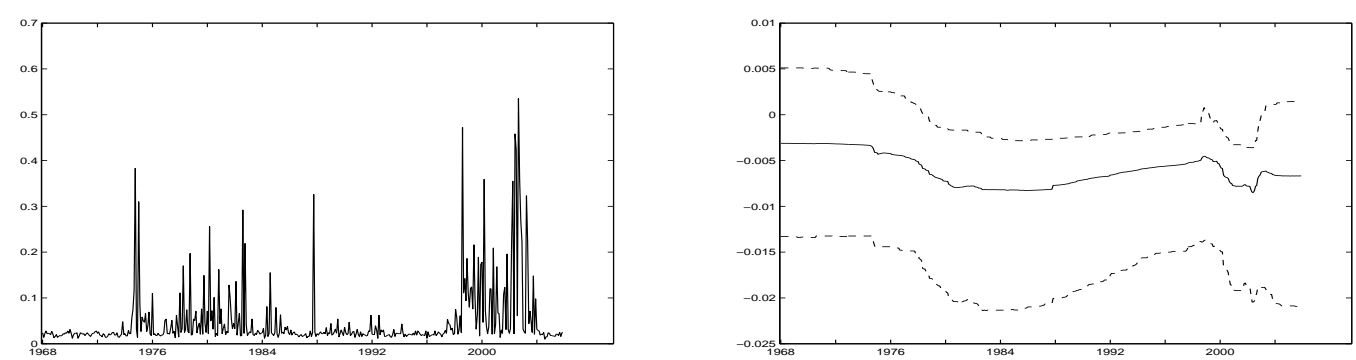

(b) $P E_{-1}$
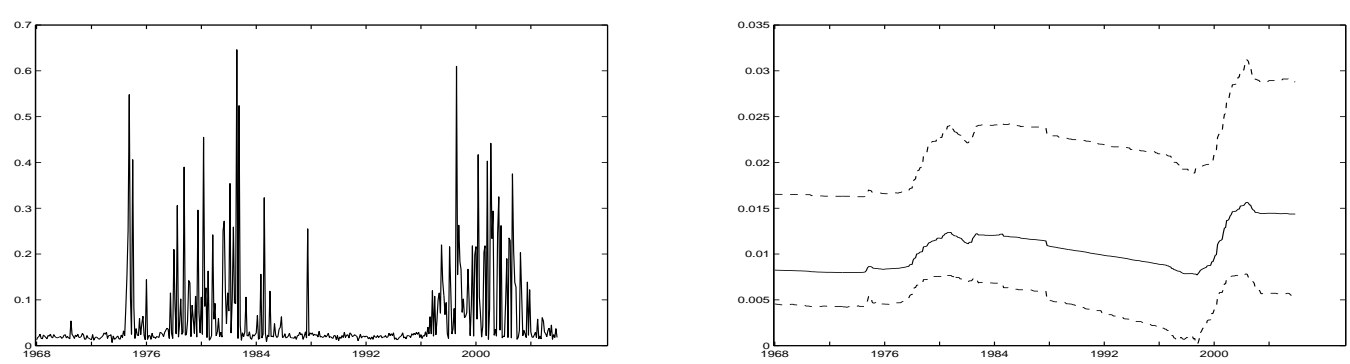

(c) $D Y_{-1}$
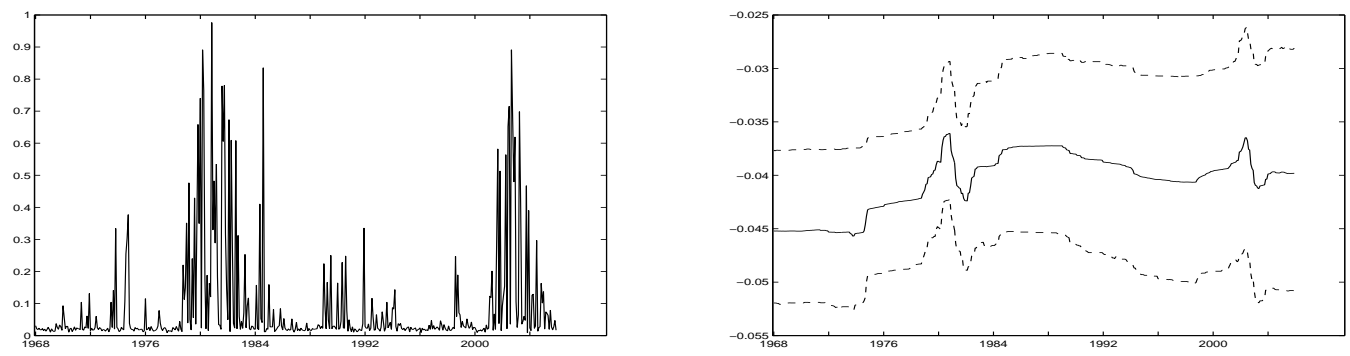

(d) $I 3_{-1}$

(continued on next page) 

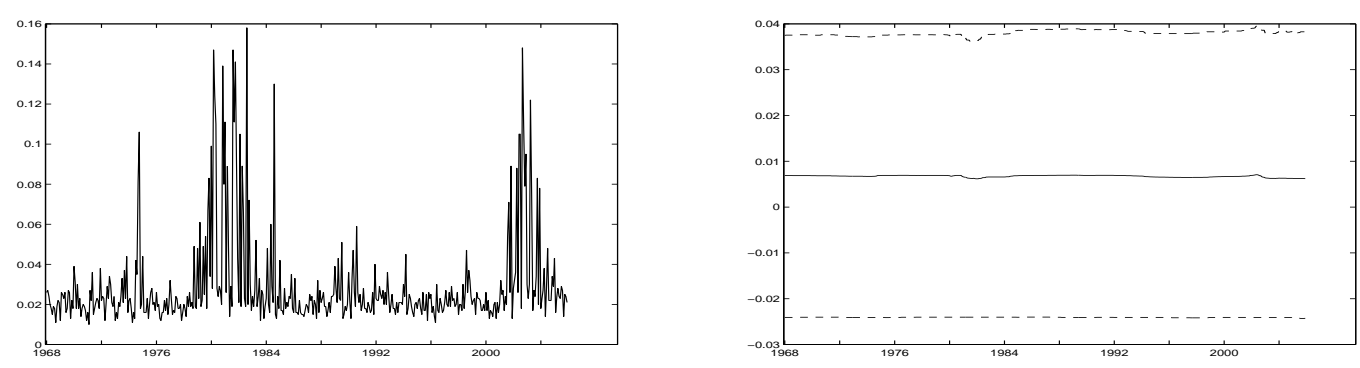

(e) $I 3_{-2}$
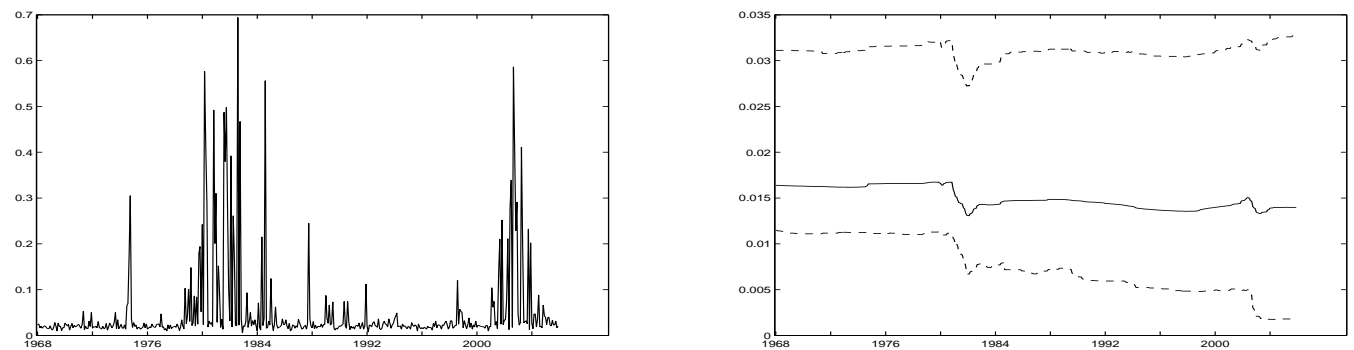

(f) $I 12_{-1}$
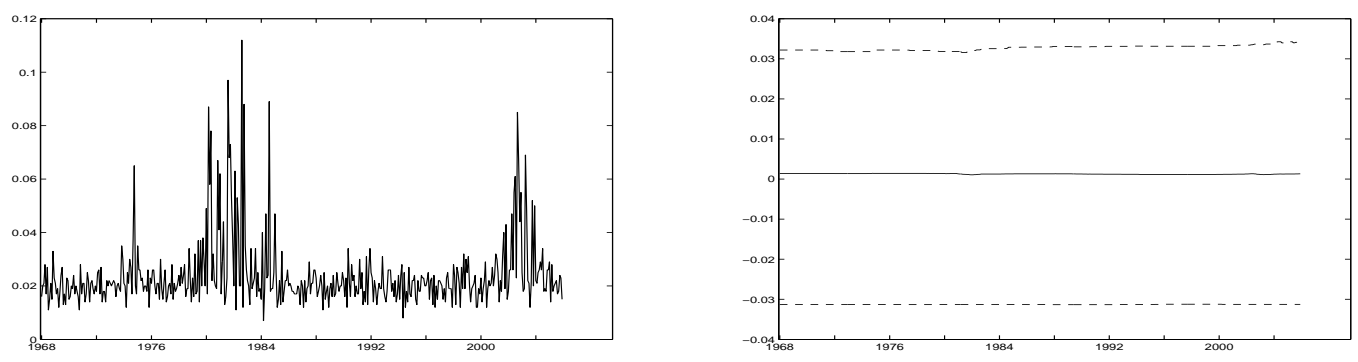

(g) $I 12_{-2}$
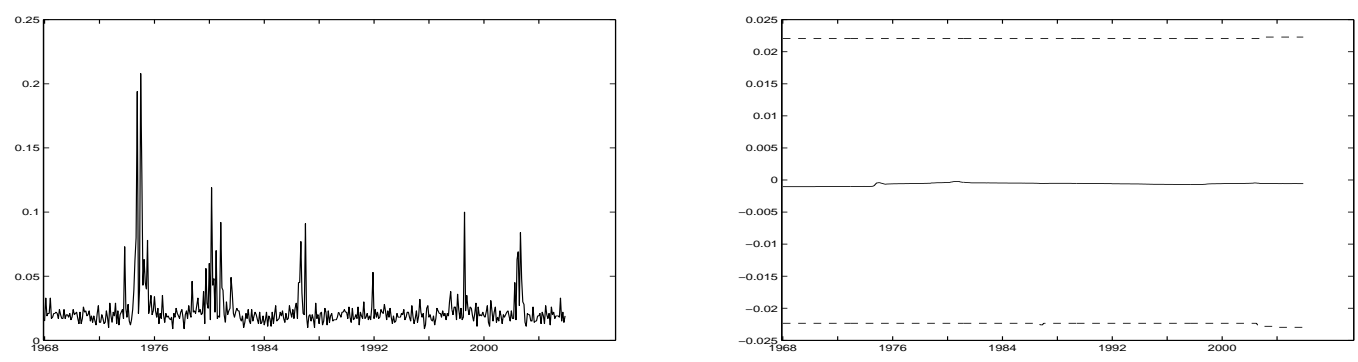

(h) $I N F_{-2}$

(continued on next page) 

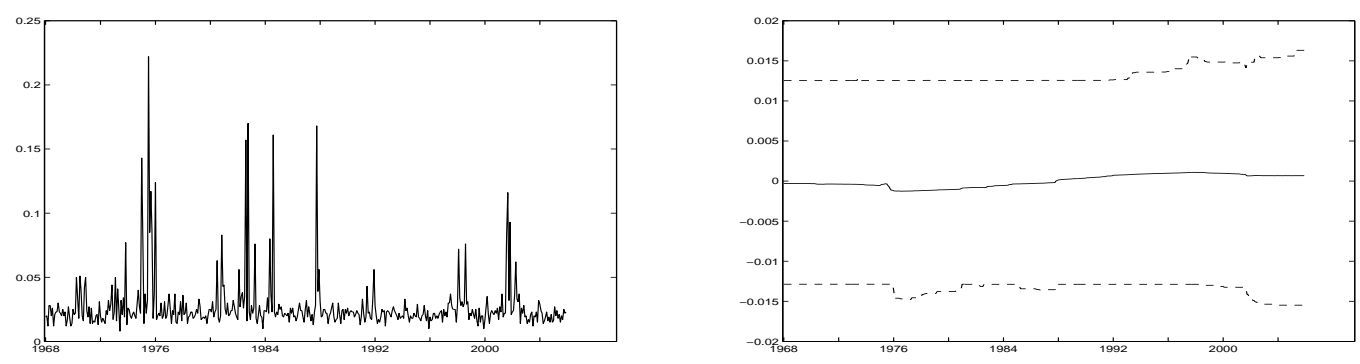

(i) $I P_{-2}$
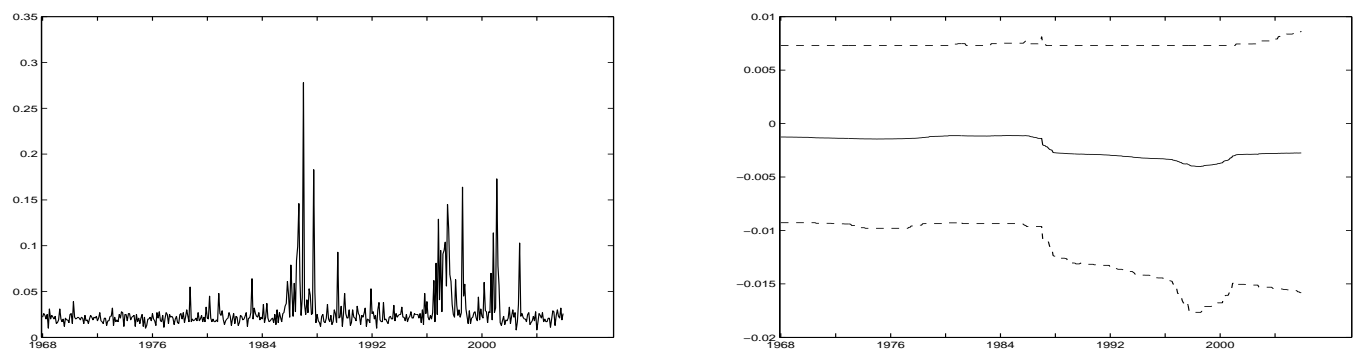

(j) $M B_{-2}$
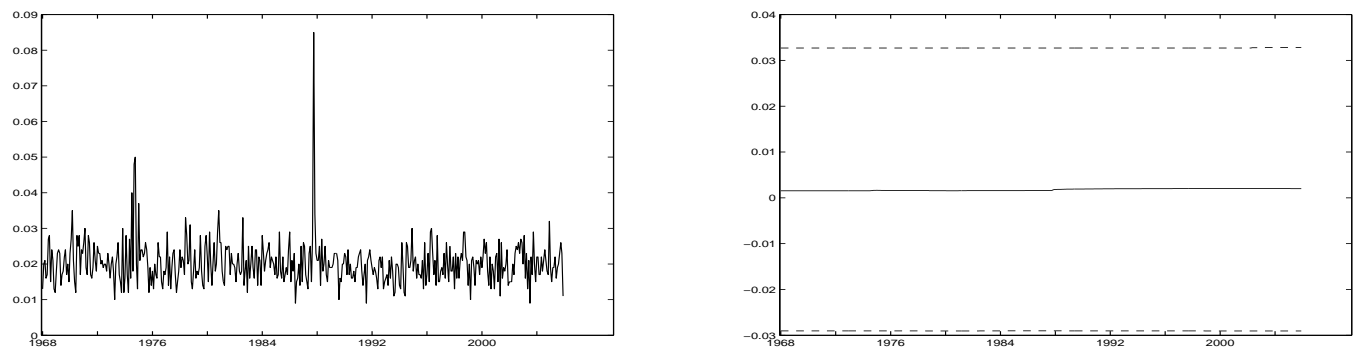

(k) $C P_{-1}$
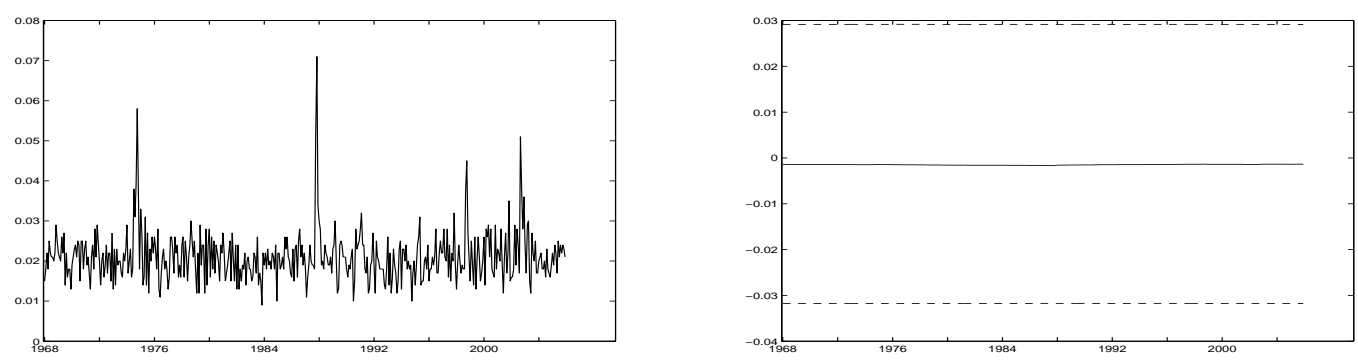

(1) $\mathrm{LVOL}_{-1}$

Note: The figure presents the posterior means (solid line) of $\kappa_{j t}$ on the left side and $\beta_{j t}$ on the right side. The dashed lines are the 25 th and 75 th percentiles of the posterior densities. 
Figure 2: Posterior densities of the breaks and $\beta$ parameters conditional on inclusion
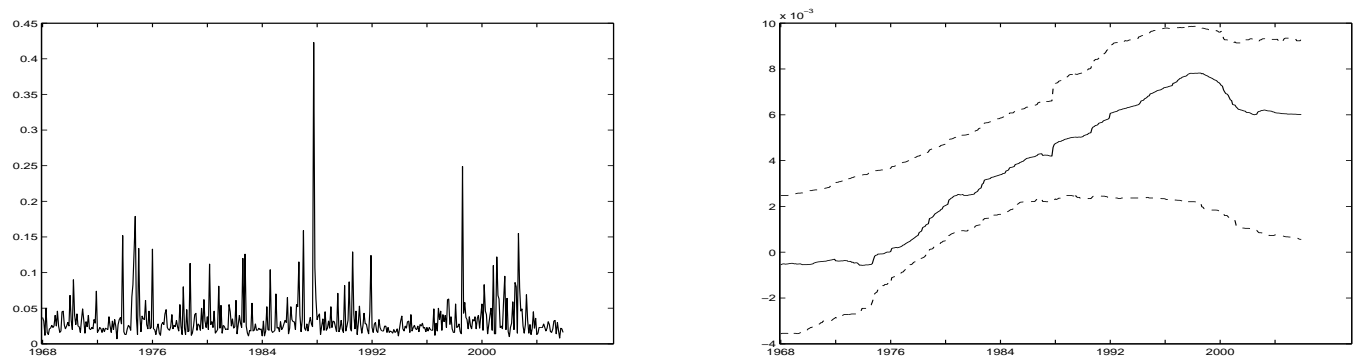

(a) Constant
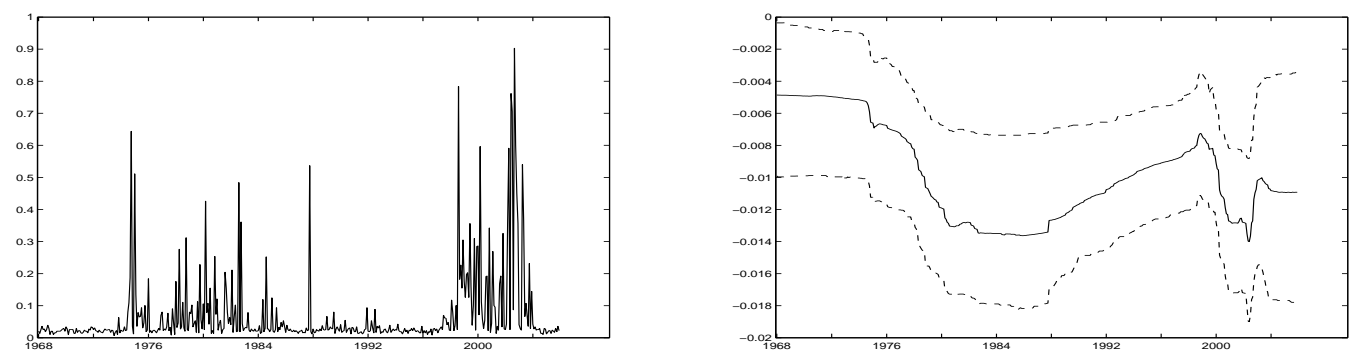

(b) $P E_{-1}$
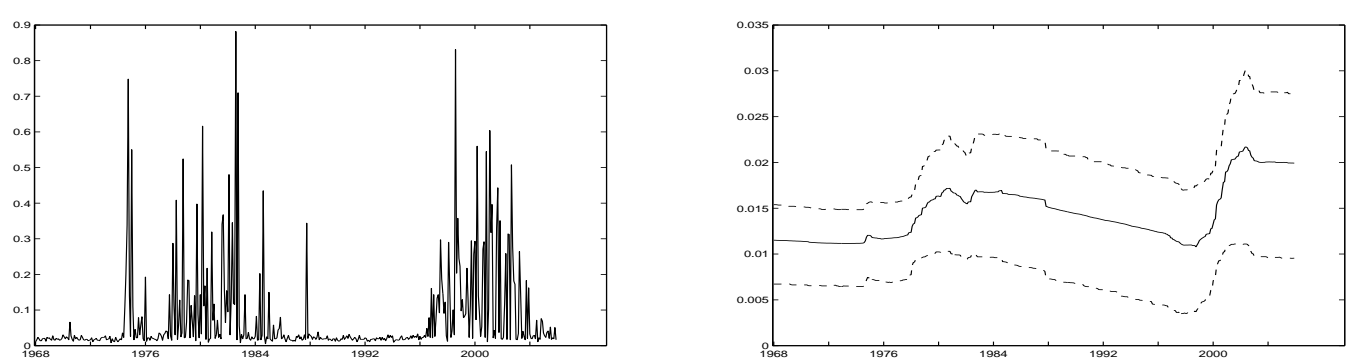

(c) $D Y_{-1}$
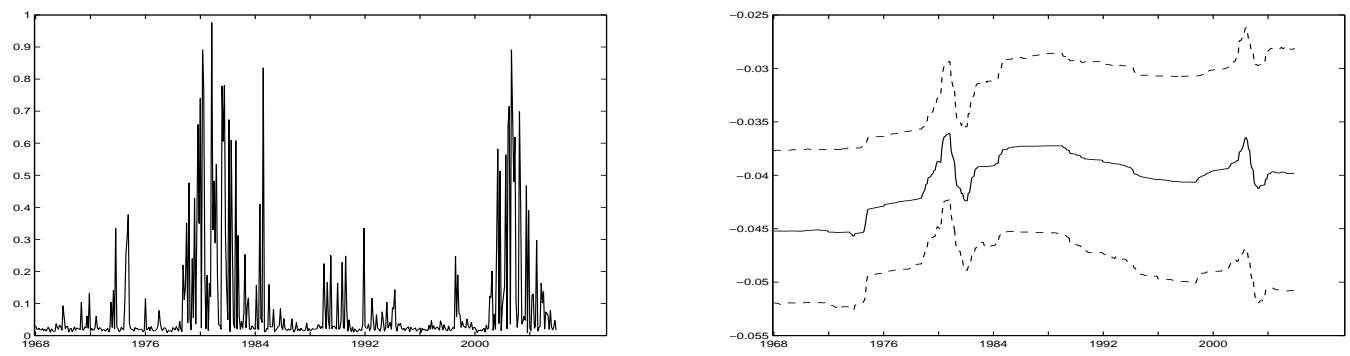

(d) $I 3_{-1}$

(continued on next page) 

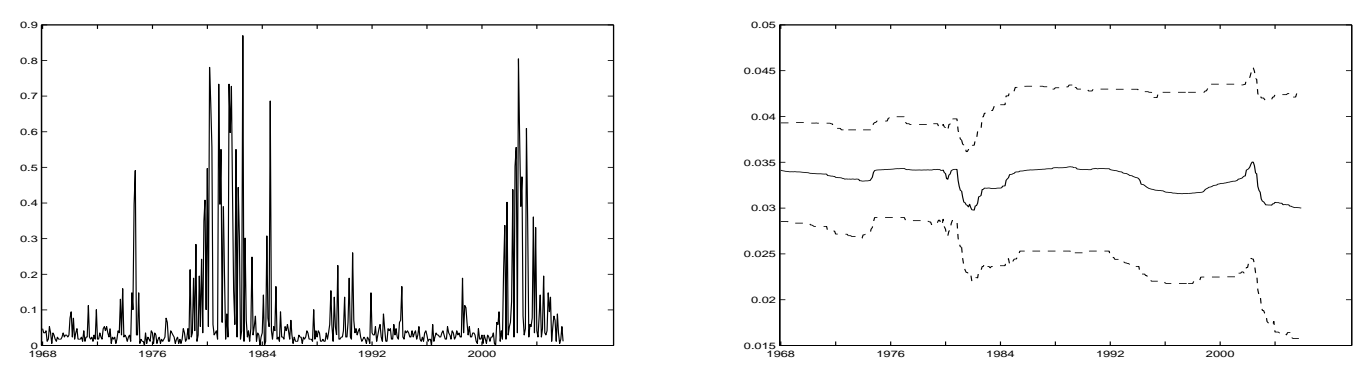

(e) $I 3_{-2}$
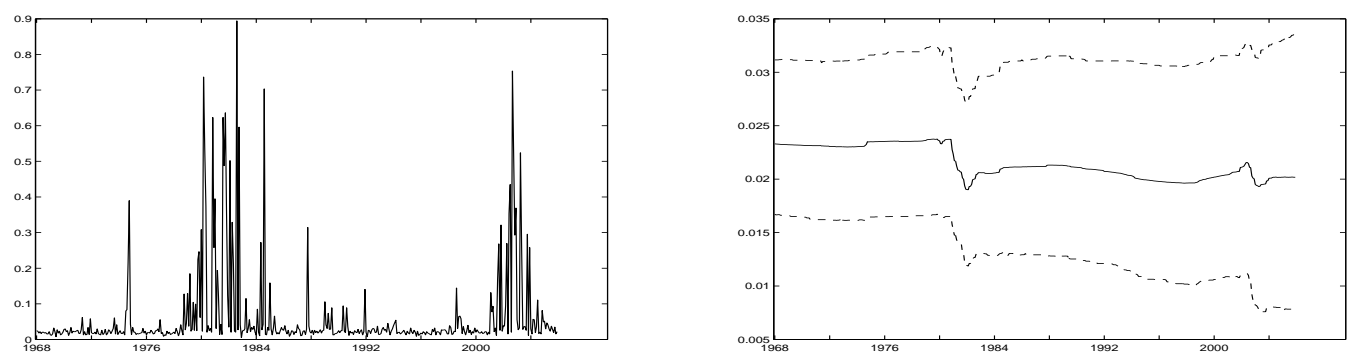

(f) $I 12_{-1}$
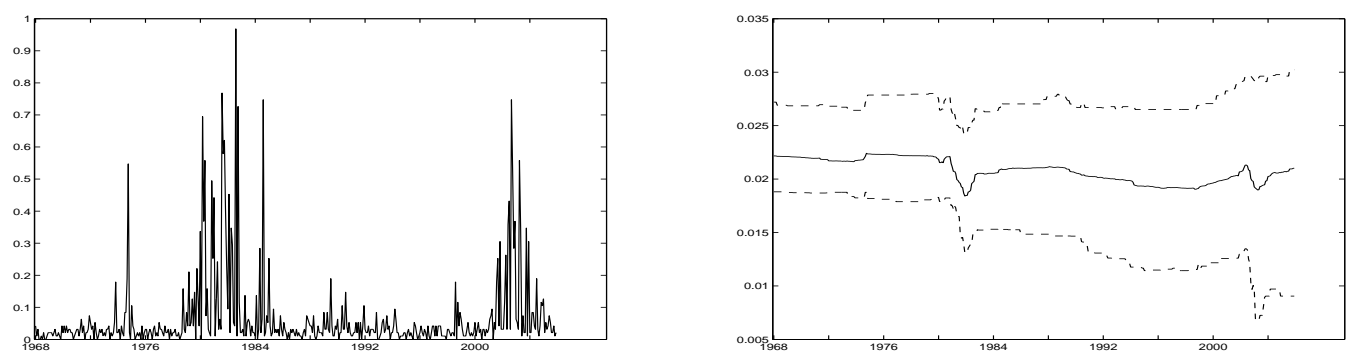

(g) $I 12_{-2}$
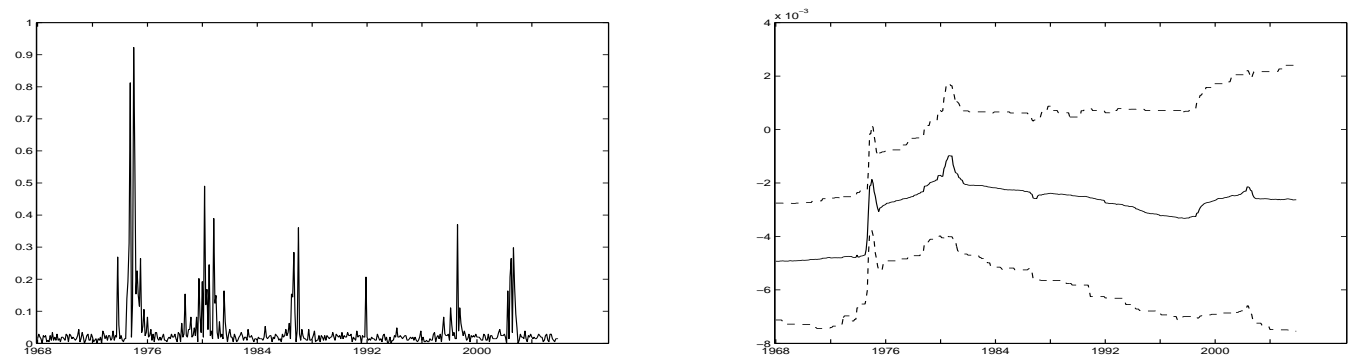

(h) $I N F_{-2}$

(continued on next page) 

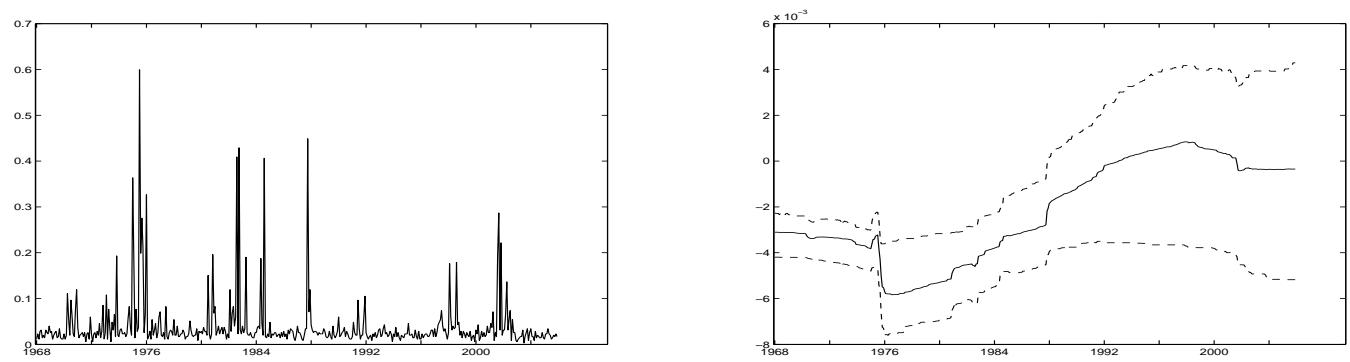

(i) $I P_{-2}$
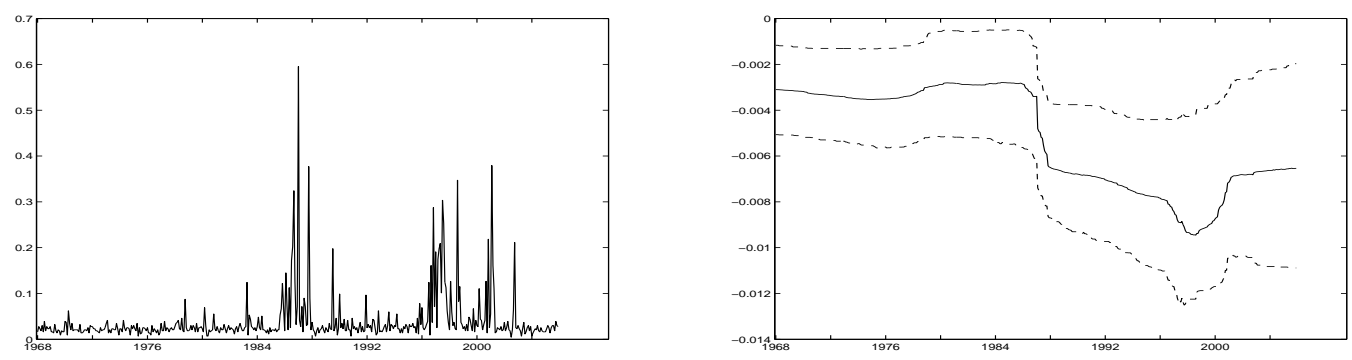

(j) $M B_{-2}$
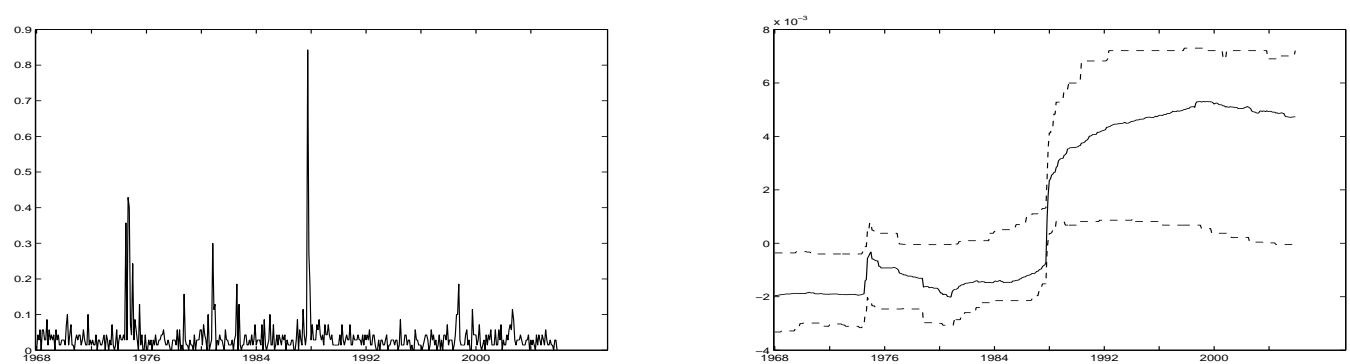

(k) $C P_{-1}$
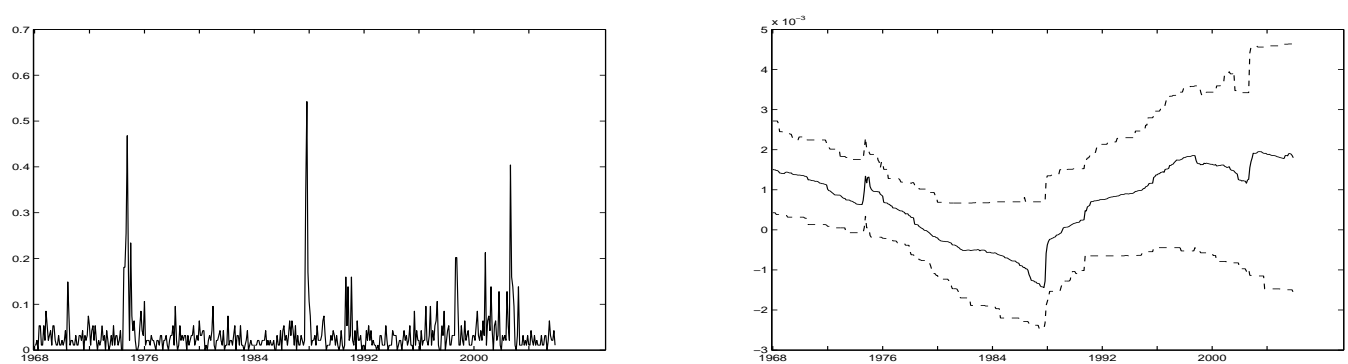

(1) $\mathrm{LVOL}_{-1}$

Note: The figure presents the posterior means (solid line) of $\kappa_{j t}$ on the left side and $\beta_{j t}$ on the right side, conditional upon inclusion of the $j$ th variable $\left(s_{j}=1\right)$. The dashed lines are the 25 th and 75 th percentiles of the posterior densities. 
Figure 3: Out-of-sample results
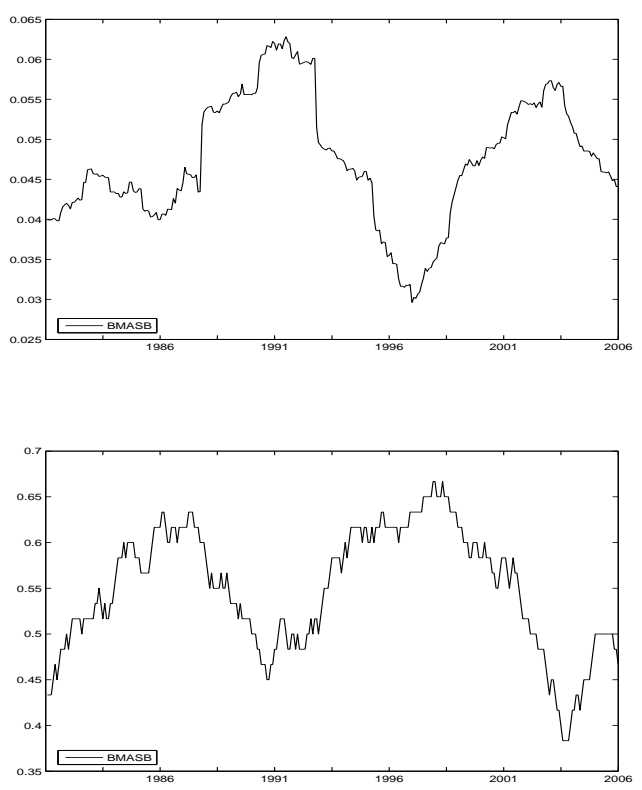

The figure presents five-year moving averages of the RMSPE on the left and of the Hit Ratio on the right. 
Figure 4: Stock portfolio weights in restricted portfolios (Strategy VII)

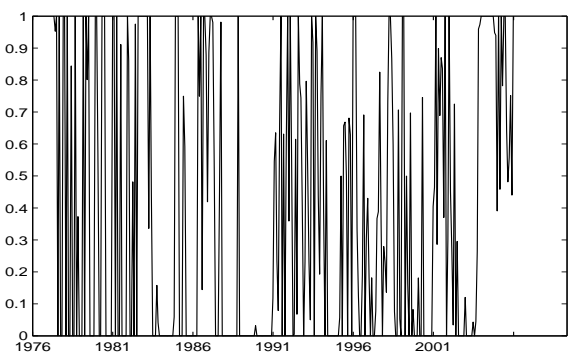

(a) $\gamma=5$

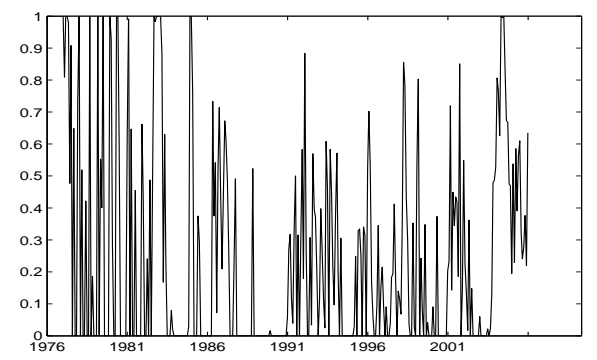

(b) $\gamma=10$

The figure presents the portfolio weight for stocks in the restricted portfolios based on excess stock return forecasts from the general model, allowing for model uncertainty and structural breaks in the regression parameters (Strategy VII). 